ARTICLE

https://doi.org/10.1038/s41467-019-09654-4

\title{
Ceramides bind VDAC2 to trigger mitochondrial apoptosis
}

\author{
Shashank Dadsena ${ }^{1,11}$, Svenja Bockelmann ${ }^{1,11}$, John G.M. Mina (1) 1,2,11, Dina G. Hassan 1,3,11, Sergei Korneev ${ }^{1}$, \\ Guilherme Razzera ${ }^{4,5}$, Helene Jahn1', Patrick Niekamp1, Dagmar Müller1, Markus Schneider1,6,7, \\ Fikadu G. Tafesse ${ }^{8}$, Siewert J. Marrink ${ }^{9}$, Manuel N. Melo (i) ${ }^{4,9}$ \& Joost C.M. Holthuis ${ }^{1,7,10}$
}

Ceramides draw wide attention as tumor suppressor lipids that act directly on mitochondria to trigger apoptotic cell death. However, molecular details of the underlying mechanism are largely unknown. Using a photoactivatable ceramide probe, we here identify the voltagedependent anion channels VDAC1 and VDAC2 as mitochondrial ceramide binding proteins. Coarse-grain molecular dynamics simulations reveal that both channels harbor a ceramide binding site on one side of the barrel wall. This site includes a membrane-buried glutamate that mediates direct contact with the ceramide head group. Substitution or chemical modification of this residue abolishes photolabeling of both channels with the ceramide probe. Unlike VDAC1 removal, loss of VDAC2 or replacing its membrane-facing glutamate with glutamine renders human colon cancer cells largely resistant to ceramide-induced apoptosis. Collectively, our data support a role of VDAC2 as direct effector of ceramide-mediated cell death, providing a molecular framework for how ceramides exert their anti-neoplastic activity.

\footnotetext{
${ }^{1}$ Molecular Cell Biology Division, Department of Biology/Chemistry, University of Osnabrück, 49076 Osnabrück, Germany. ${ }^{2}$ School of Science, Engineering and Design, Teesside University, Middlesbrough TS1 3BX, UK. ${ }^{3}$ Institute of Environmental Studies and Research, Ain Shams University, Cairo, Egypt. ${ }^{4}$ Instituto de Tecnologia Química e Biológica António Xavier, Universidade Nova de Lisboa, Av. da República, 2780-157 Oeiras, Portugal. ${ }^{5}$ Departamento de Bioquímica, Centro de Ciências Biológicas, Universidade Federal de Santa Catarina, Florianópolis, Brazil. ${ }^{6}$ Plant Physiology Division, Department of Biology/ Chemistry, University of Osnabrück, 49076 Osnabrück, Germany. ${ }^{7}$ Center for Cellular Nanoanalytics, Osnabrück University, Artilleriestraße 77,49076 Osnabrück, Germany. ${ }^{8}$ Molecular Microbiology and Immunology, Oregon Health \& Science University, Portland, OR 97239, USA. ${ }^{9}$ Groningen Biomolecular Sciences and Biotechnology Institute and Zernike Institute for Advanced Materials, University of Groningen, Nijenborgh 7, 9747 AG Groningen, The Netherlands. ${ }^{10}$ Membrane Biochemistry and Biophysics, Bijvoet Center and Institute of Biomembranes, Utrecht University, $3584 \mathrm{CH}$ Utrecht, The Netherlands. ${ }^{11}$ These authors contributed equally: Shashank Dadsena, Svenja Bockelmann, John G. M. Mina, Dina G. Hassan. Correspondence and requests for materials should be addressed to J.G.M.M. (email: j.mina@tees.ac.uk) or to M.N.M. (email: m.n.melo@itqb.unl.pt) or to J.C.M.H. (email: holthuis@uos.de)
} 
S phingolipids are essential components of eukaryotic membranes that participate in a broad range of cellular processes by controlling vital physical membrane properties and as signaling molecules in response to physiological cues and stres$\operatorname{ses}^{1-3}$. Notably ceramides, the central intermediates of sphingolipid metabolism, have emerged as key mediators of antiproliferative and tumor suppressive cellular programs such as apoptosis, mitophagy, cell cycle arrest, and senescence ${ }^{4,5}$. Multiple stress stimuli, including tumor necrosis factor a $(\mathrm{TNF} \alpha)^{6,7}$, ionizing radiation ${ }^{8,9}$, and chemotherapeutic drugs ${ }^{10,11}$, cause a rise in ceramide levels through stimulation of de novo ceramide synthesis, activation of sphingomyelin hydrolysis, or both. Interventions that suppress ceramide accumulation render cancer cells resistant to these stress-inducing agents, indicating that ceramides are bona fide anti-proliferative and pro-apoptotic signaling molecules. While these findings raised considerable interest in targeting sphingolipid-metabolizing enzymes for cancer therapy $5,12,13$, the mechanisms by which ceramides execute their tumor-suppressive activities are incompletely understood.

Mitochondria serve a central role in apoptosis induced by stress stimuli. The mitochondria from cancer cells are often resistant to induction of mitochondrial outer membrane permeabilization (MOMP), a point of no return in the intrinsic pathway of apoptosis. MOMP allows the passage of intermembrane space proteins such as cytochrome $c$ to activate caspases, a family of cysteine proteases responsible for executing an ordered destruction of the cell $^{14}$. MOMP is controlled by pro- and anti-apoptotic members of the B-cell lymphoma $2(\mathrm{Bcl}-2)$ protein family, which collectively determine the balance between cell death and survival ${ }^{15,16}$. The main function of the anti-apoptotic $\mathrm{Bcl} 2$ proteins is to counter the pro-apoptotic activities of the Bcl-2 proteins Bax and Bak, which directly mediate MOMP by creating proteolipid pores responsible for cytochrome $c$ release ${ }^{17,18}$. Several reports have indicated that ceramides can trigger MOMP by modulating the activity of kinases or phosphatases implicated in controlling Bcl-2 protein function. In cells, elevated ceramide levels have been shown to inhibit phosphoinositide-3-kinase (PI3K) and Akt/PBK signaling, resulting in dephosphorylation and subsequent activation of proapoptotic Bcl-2-family protein $\mathrm{Bad}^{19,20}$. Short-chain ceramides can bind and stimulate protein phosphatase 2A (PP2A), which dephosphorylates and inactivates the anti-apoptotic protein BCL2 21,22 .

Other studies revealed that ceramides can also act directly on mitochondria to trigger MOMP and apoptotic cell death ${ }^{23}$. For instance, mitochondrial targeting of a bacterial sphingomyelinase to generate ceramides in mitochondria or directing CERTmediated ceramide transport to mitochondria induces cytochrome $c$ release and apoptosis ${ }^{24,25}$. In addition, ER-like membranes associated with isolated mitochondria appear to produce sufficient amounts of ceramides to enable a transient passage of cytochrome $c$ across the outer membrane ${ }^{26}$. However, the underlying mechanisms remain to be established. Interestingly, ceramides have been shown to form pores in model bilayers as well as in the outer membrane of isolated mitochondria that are large enough to mediate passage of cytochrome $c^{27,28}$. Formation of ceramide channels does not rely on any particular protein but is disrupted by anti-apoptotic Bcl-2 proteins ${ }^{29}$. It has also been suggested that ceramides accumulating in the mitochondrial membrane of mammalian cells upon irradiation form or stabilize microdomains that serve as platforms into which Bax inserts and assembles into an active pore ${ }^{30}$. While ceramides have been proposed to cooperate directly with Bax in the assembly of cytochrome $c$ conducting channels ${ }^{30,31}$, other experiments with isolated mitochondria suggest that metabolic conversion of ceramides into sphingosine-1-phosphate and hexadecenal is necessary to facilitate Bax/Bak activation leading to $\mathrm{MOMP}^{32}$.
In this study, we present evidence for an alternative mechanistic view, namely that ceramides mediate their pro-apoptotic activity at least in part by interacting directly and specifically with the voltage-dependent anion channel VDAC2, a mitochondrial platform for Bax/Bak translocation ${ }^{33-35}$. Identification of VDAC2 as an effector of ceramide-mediated cell death provides new opportunities for exploiting the therapeutic potential of ceramides as tumor suppressor lipids.

\section{Results}

A chemical screen for ceramide-binding proteins yields VDACs. To identify proteins involved in ceramide-mediated stress signaling and apoptosis, we used a bifunctional ceramide analog carrying a photoactive diazirine and clickable alkyne group in its $N$-linked acyl chain (pacCer, Fig. 1a) ${ }^{36}$. Total membranes from human HeLa cells were incubated with pacCercontaining liposomes, subjected to UV crosslinking and click reacted with Alexa Fluor 647 azide $\left(A F 647-N_{3}\right)$. In-gel fluorescence (IGF) analysis revealed a subset of membrane-bound proteins with affinity for the pacCer probe, which included a mitochondria-associated protein of $\sim 33 \mathrm{kDa}$ that was prominently photolabeled (Fig. 1b, Supplementary Fig. 1). As ceramide exerts its apoptogenic activity in mitochondria ${ }^{8,24,25}$, we set out to identify the $33 \mathrm{kDa}$ candidate ceramide-binding protein (CBP). To this end, mitochondria were photolabeled with pacCer and then click reacted with a PEG-based reagent containing an azide, a biotin and a TAMRA fluorophore as functional groups (Fig. 1c). Next, pacCer-crosslinked proteins were isolated using NeutrAvidin agarose and visualized by IGF (Fig. 1d). The fluorescent $33 \mathrm{kDa}$ protein band was cut from the gel, trypsin-digested, and identified by LC-MS/MS analysis as the voltage-dependent anion channel isoforms VDAC1 and VDAC2 (Supplementary Table 1). In line with the MS data, pretreatment of HeLa cells with VDAC1 and VDAC2-targeting siRNAs effectively depleted the fluorescent $33 \mathrm{kDa}$ protein band from pacCer-labeled and Alexa click-reacted mitochondria (Fig. 1e, f). The photolabeled $33 \mathrm{kDa}$ protein band also cross-reacted with both anti-VDAC1 and anti-VDAC2 antibodies (Fig. 1g, h). In contrast, VDAC3 and TOM40-an outer mitochondrial membrane (OMM) channel protein with a cellular copy number close to that of $\mathrm{VDACs}^{37}$-both lacked affinity for pacCer, indicating that VDAC1 and VDAC2 are genuine mitochondrial CBPs.

MD simulations uncover a ceramide-binding site on VDACs. To search for a putative ceramide-binding site on VDAC1 and VDAC2, we performed coarse-grain molecular dynamics (CGMD) simulations using the Martini model ${ }^{38-40}$. Main simulations were performed with VDAC channels at an aggregate time of $1.23 \mathrm{~ms}$ (Supplementary Table 2) —only attainable using CG-MD. A well-resolved structure of mouse VDAC1 (PDB: 4C69) ${ }^{41}$ was used as a base template. An available structure of VDAC2 from zebrafish (PDB:4BUM) ${ }^{42}$ showed almost perfect structural identity to VDAC1 ( $1.7 \AA$ barrel backbone RMSD). From the assumption of identical secondary structure we mutated VDAC1 side chains to the mouse sequences of VDAC2 and VDAC3 to obtain all three isoforms for comparison. A bilayer mimicking the $\mathrm{OMM}^{43}$ was constructed with $\sim 630$ lipids. To the outer leaflet of this bilayer, 16 molecules of $\mathrm{C}_{16}$-ceramide were added. In agreement with the photolabeling data, simulations revealed that VDAC1 and VDAC2, but not VDAC3, have a binding site for ceramide buried in the membrane interior on one side of the barrel wall, comprising $\beta$-strands 3-5 (Fig. 2a). This site harbors a uniquely positioned glutamate (Glu) residue in the transmembrane region of $\beta$-strand $4-$ Glu73 in VDAC1 and Glu84 in VDAC2-that faces the bilayer's hydrophobic core 
a

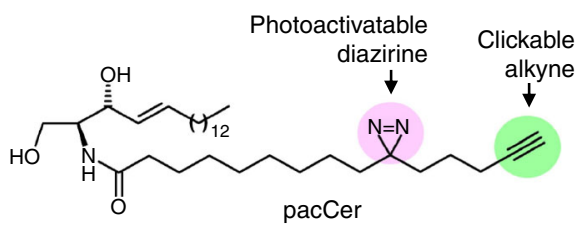

C

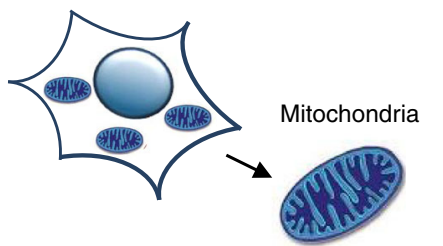

b

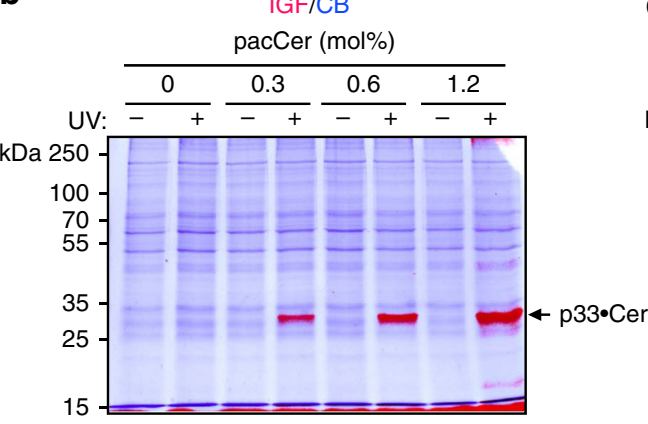

d

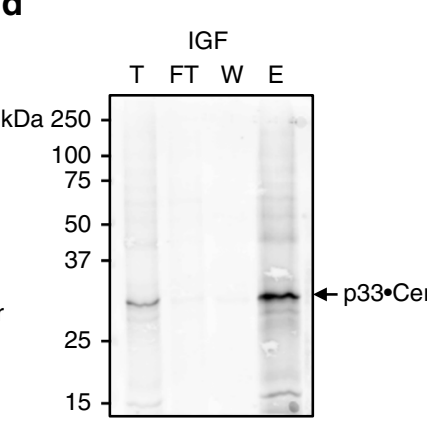

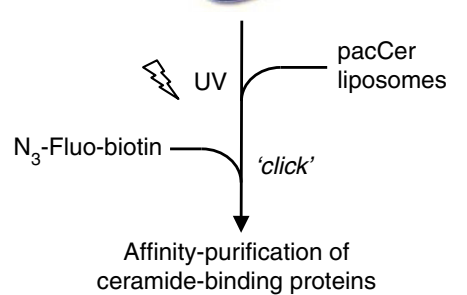

$\forall$

MS analysis

$\downarrow$

VDAC $1 / 2$ e

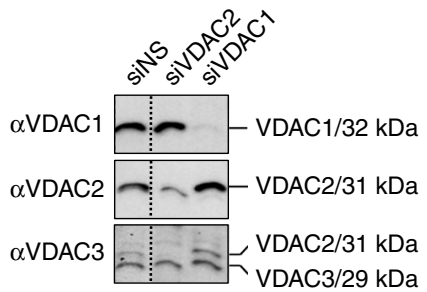

f $\mathbf{g}$

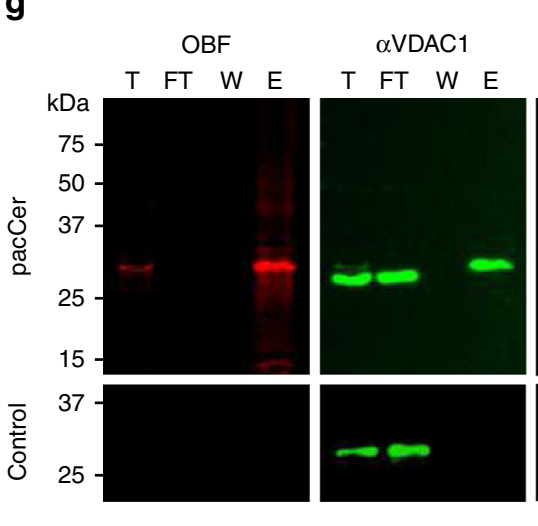

ap60-Mito/ ap60-Mito/ Merge T
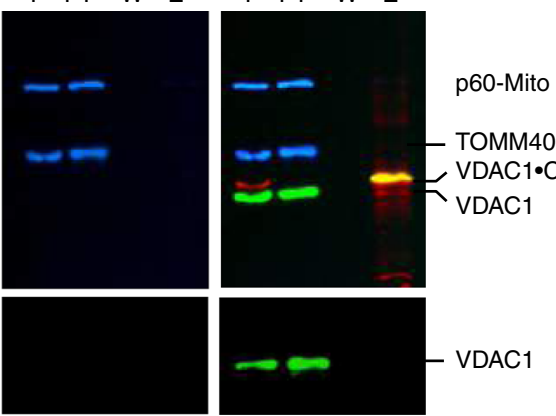

h

OBF $\quad \alpha$ VDAC2

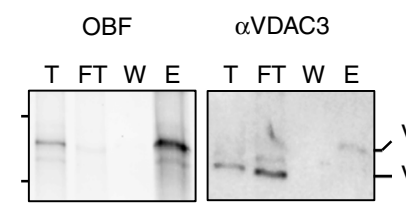
VDAC2・Cer

Fig. $1 \mathrm{~A}$ chemical screen for mitochondrial ceramide-binding proteins yields VDAC1 and -2. a Structure of the photoactive and clickable $\mathrm{C}_{15}$-ceramide analog, pacCer. b Mitochondria isolated from HeLa cells were incubated with liposomes containing increasing amounts of pacCer, UV irradiated, and then click reacted with AF647- $\mathrm{N}_{3}$. Samples were processed for SDS-PAGE, subjected to in-gel fluorescence (IGF, red), and stained with Coomassie blue (CB, blue). p33•Cer denotes a prominently photolabeled protein band of $\sim 33 \mathrm{kDa}$. c Strategy for the identification of p33•Cer. $\mathbf{d}$ p33•Cer was purified from pacCer-labeled and TAMRA/biotin click-reacted mitochondria using NeutrAvidin-beads, imaged by IGF, excised from the gel, digested by trypsin, and then identified by LC-MS/MS. Data from two independent experiments revealed that p33•Cer corresponds to VDAC1 and VDAC2. e Specificity of anti-VDAC antibodies was validated by immunoblotting of mitochondria isolated from HeLa cells treated with non-silencing (siNS) or VDAC-targeting siRNAs (siVDAC1, siVDAC2). Note that the anti-VDAC3 antibody cross-reacts with VDAC2. $f$ Mitochondria isolated from siVDAC1/2-treated HeLa cells were photolabeled with pacCer, click-reacted with $\mathrm{AF} 647-\mathrm{N}_{3}$, and subjected to IGF analysis followed by CB staining. $\mathrm{g}$ Fractions obtained during affinity purification of $\mathrm{p} 33 \cdot \mathrm{Cer}$ were subjected to SDS-PAGE, transferred on nitrocellulose, analyzed by on-blot-fluorescence (OBF) and probed with antibodies against VDAC1, TOM40, and p60-Mito. $\mathbf{h}$ Fractions obtained during affinity purification of p33•Cer were processed as in $\mathbf{g}$ and probed with anti-VDAC2 and anti-VDAC3 antibodies. T total mitochondria extract, FT flow-through, W wash, E eluate

(Fig. 2b). In its deprotonated state, this residue seemed to promote direct contact with the ceramide head group (Fig. 2c; Supplementary Information Videos 1 and 2). In VDAC3, which does not bind ceramide (Figs. $1 \mathrm{~h}$ and $2 \mathrm{a}$ ), the bilayer-facing Glu residue is replaced by a glutamine (Gln73; Fig. 2b). Substitution of Gln for Glu73 in VDAC1 or Glu84 in VDAC2 strongly reduced the ceramide occupancy and residence time at the binding sites (Fig. 2d, e). Protonation of the bilayer-facing Glu also greatly diminished ceramide-binding (Fig. 3a, b) while substitution of a deprotonated aspartate for the Glu residue retained ceramide binding (Supplementary Fig. 2). This indicates that a negative charge on the membrane-buried Glu residue is critical for ceramide binding.

In line with a previous computational study ${ }^{44}$, we also found a number of binding sites for cholesterol. These displayed no overlap with the ceramide-binding site (Fig. 2d). To exclude the possibility that competition with ceramide prevented cholesterol to occupy the ceramide-binding site, additional simulations were 
a

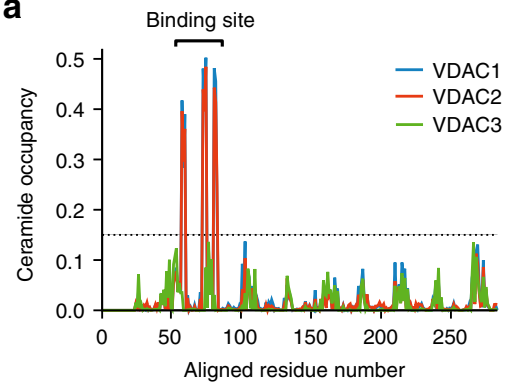

C

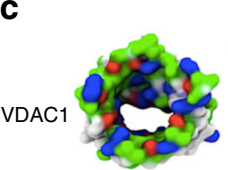

Glu73
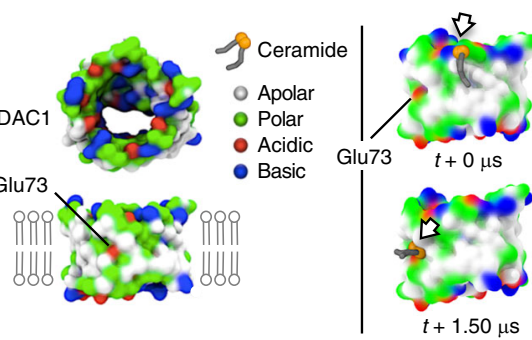

b

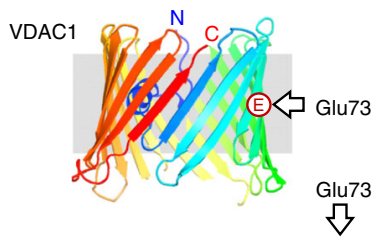

VDAC1 52 TKVTGSLETKYRWTEYGLTFTEKWNTDNTLGTEITVEDQ 90 VDAC2 63 GKVTGTLETKYKWCEYGLTFTEKWNTDNTLGTEIAIEDQ 101 VDAC3 52 GKASGNLETKYKVCNYGLTFTQKWNTDNTLGTEISWENK 90

$\beta 4$

$\beta 5$

e
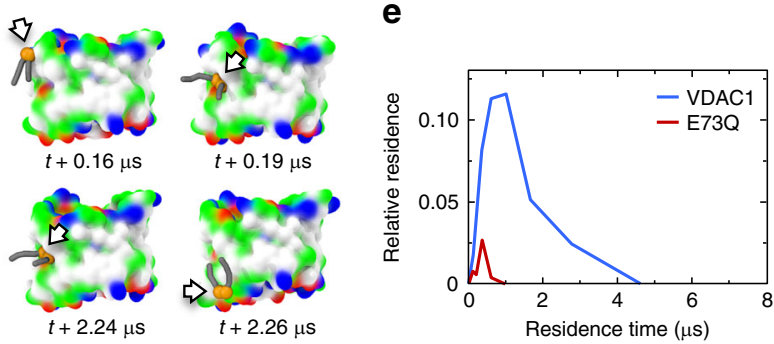

d

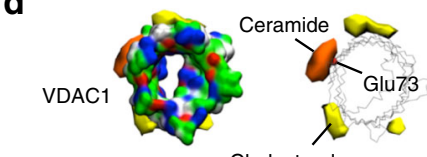

VDAC1 ${ }^{\mathrm{E} 30}$ ats

$t+2.24 \mu \mathrm{s}$

$t+2.26 \mu \mathrm{s}$
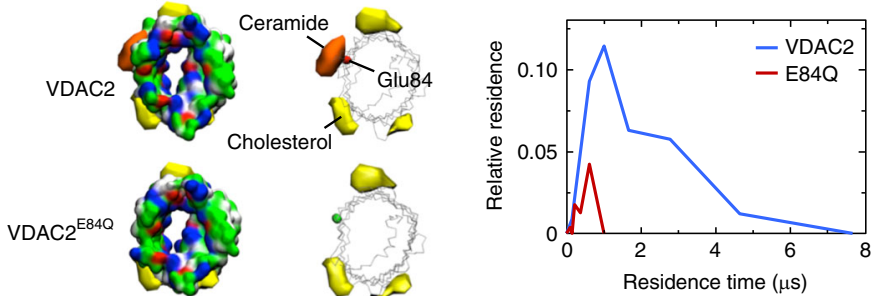

Fig. 2 MD simulations uncover a putative ceramide-binding site on VDAC1 and -2. a Ceramide head group contact occupancy of mouse VDAC1, VDAC2, and VDAC3 in an OMM model containing 5 mol\% ceramide, with 1.0 corresponding to a ceramide contact during the entire simulation time. A threshold of $15 \%$ occupancy, based on the occupancies of non-binding site residues, is indicated by a dotted blue line. VDAC1 and VDAC2 have a clear ceramidebinding site, comprising residues 58-62, 71-75, and 81-85; this site is lacking in VDAC3. b Sequence alignment revealing the position of a bilayer-facing Glu residue in VDAC1 (E73) and VDAC2 (E84), which is replaced by GIn in VDAC3 (Q73). c Stills from an MD simulation, showing the approach and binding of a ceramide molecule to VDAC1 in close proximity of the bilayer-facing Glu residue in its deprotonated state. Protein surface colors mark polar (green), apolar (white), cationic (blue), or anionic (red) residues. d Space-filling and wireframe models of VDAC1, VDAC1E73Q, VDAC2, and VDAC2 E84Q with deprotonated E73/E84. Indicated are the volumes for which there is ceramide occupancy greater than 10\% (orange) or cholesterol occupancy greater than $20 \%$ (yellow). e Distribution of the durations of ceramide contacts with VDAC1, VDAC1E73Q, VDAC2, and VDAC2 ${ }^{\mathrm{E} 84 \mathrm{Q}}$ at the preferred binding site as in $\mathbf{d}$. The $y$-axis indicates the fraction of the total system time spent in binding events of the duration indicated by $x$. Summing all points' $y$-values yields the fraction of total simulation time when ceramide was bound

performed in the absence ceramide. Also in those simulations no cholesterol binding near the membrane-facing Glu was observed (Supplementary Fig. 3). We observed zero-specific binding events between phosphatidylcholine (PC) and VDACs in the OMM mimics. Yet when VDAC1 was simulated in a bilayer of $100 \%$ dimyristoyl-phosphatidylcholine (DMPC) following the setup of a previous atomic resolution simulation analysis ${ }^{45}$, contacts of the PC head group with the bilayer-facing deprotonated Glu residue could occasionally be observed (Supplementary Fig. 4a). However, these encounters were much shorter-lived ( $\leq 5 \mathrm{~ns}$; Supplementary Fig. 4b) and extremely rare in comparison to those involving ceramide, which displayed average residence times of 0.8 and $1.2 \mu$ s for VDAC1 and VDAC2, respectively (Supplementary Fig. 4c). In sum, CG-MD simulations revealed that VDAC1 and VDAC2 each harbor a binding site for ceramide, with a charged Glu residue buried in the membrane interior serving a critical role in ceramide binding.

Ceramide binding relies on a membrane-facing glutamate. We next sought to verify the relevance of the membrane-facing Glu residue in VDACs for ceramide binding. To this end, human
VDAC1 and VDAC2 and the mutant channels VDAC1 ${ }^{\mathrm{E} 73 \mathrm{Q}}$ and VDAC2 ${ }^{\mathrm{E} 84 \mathrm{Q}}$ were produced recombinantly in $E$. coli and then reconstituted in egg PC liposomes (Supplementary Fig. 5). Density gradient fractionation analysis revealed that reconstitution efficiencies of wild type and mutant channels were practically indistinguishable. The reconstituted channels were then subjected to photolabeling with pacCer and bifunctional analogs of diacylglycerol (pacDAG), PC (pacPC), phosphatidylethanolamine (pacPE), and cholesterol (pacChol; Supplementary Fig. 6). VDAC1 and VDAC2 could be efficiently and reproducibly photolabeled with pacCer, pacPC, and pacChol, but not with pacDAG or pacPE (Fig. 4). In agreement with the simulations, replacing the membrane-facing Glu with Gln virtually abolished labeling of both channels with pacCer and pacPC, whereas labeling with pacChol was not or only slightly affected (Figs. 4 and $5 \mathrm{a}$ ). Moreover, reducing the $\mathrm{pH}$ from 7 to 5 caused a significant reduction in E73-dependent photolabeling of VDAC1 with pacCer (Fig. 3c, d). This suggests that ceramide binding is critically dependent on the protonation state of the membraneexposed Glu, as predicted by the simulations.

The pronounced labeling of the wild-type channels with pacPC was somewhat unexpected as simulations revealed that specific 
a

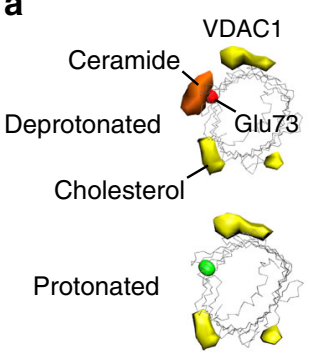

b
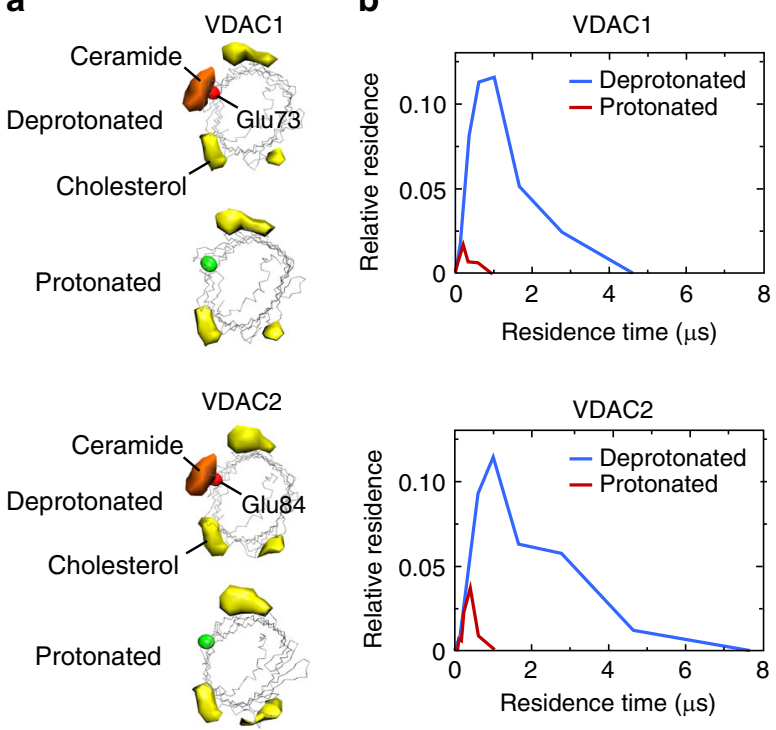

C

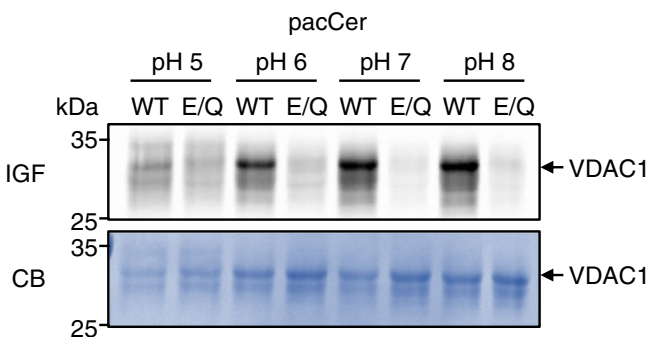

d

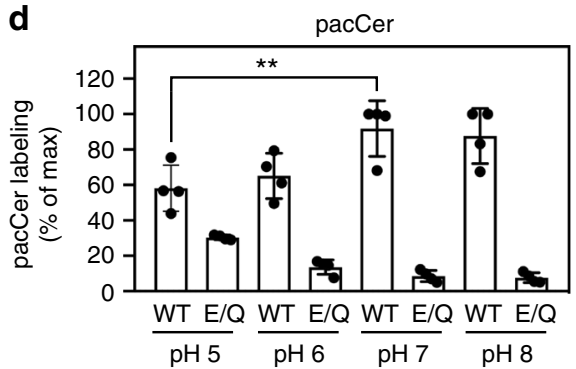

Fig. 3 Ceramide binding by VDACs relies on the protonation state of the bilayer-facing Glu. a Space-filling and wireframe models of VDAC1 and VDAC2 simulated with the bilayer-facing Glu residue in a protonated or deprotonated state. Indicated are the volumes for which there is ceramide occupancy greater than $10 \%$ (orange) or cholesterol occupancy greater than $20 \%$ (yellow). b Distribution of the durations of ceramide contacts with VDAC1 and VDAC2 at the preferred binding site as in $\mathbf{a}$. The $y$-axis indicates the fraction of the total system time spent in binding events of the duration indicated by $x$. Summing all points' $y$-values yields the fraction of total simulation time when ceramide was bound. c Human VDAC1 and VDAC1E73Q were produced in E. coli, purified, reconstituted in liposomes, and then photolabeled at the indicated pH with pacCer added from an ethanolic stock. Samples were click-reacted with AF647- $\mathrm{N}_{3}$, subjected to SDS-PAGE, and analyzed by IGF and CB staining. $\mathbf{d}$ Quantitative analysis of relative pacCer photolabeling efficiencies of reconstituted VDAC1 treated as in $\mathbf{c}$. Data are means \pm s.d.; $n=4 ;{ }^{\star \star} p<0.01$ by two-tailed paired $t$-test. Source data

encounters of PC molecules with the membrane-facing Glu are relatively rare. This discrepancy could be due to the irreversible nature of photoaffinity labeling in combination with the major difference in timescales between CG-MD simulations ( $\mu$ s range) and photoaffinity labeling (second range). While our data revealed no obvious overlap between the ceramide and cholesterol binding sites on VDACs, a recent photolabeling study mapped the bilayer-facing Glu to a major cholesterol binding pocket on $\mathrm{VDACl}^{46}$. In contrast to the present work, the latter study was performed on VDAC1-containing bicelles with cholesterol probes that carry the photoactive diazirine in the aliphatic tail or at C7. Thus, additional work will be necessary to resolve the discrepancy between the simulations and photolabeling studies of cholesterol binding to VDACs.

As aliphatic diazirines display photochemical preference for nucleophilic amino acids ${ }^{47}$, the deprotonated side chain of the membrane-buried Glu in VDACs may provide a site of insertion for the diazirine in pacCer, which may diffuse a short distance to reach such a nucleophile. Therefore, we next labeled VDACs with pacCer in the presence of excess $\mathrm{C}_{16}$-ceramide. Labeling by pacCer was progressively reduced by $\mathrm{C}_{16}$-ceramide when added in 3- to 27-fold excess (Fig. 5b), arguing against the idea that pacCer labeling of VDACs is primarily driven by affinity of the aliphatic diazirine for the negatively charged Glu. Excess $\mathrm{C}_{16^{-}}$ ceramide did not affect labeling of VDACs with pacChol. In line with the simulations, these data indicate that ceramides bind VDACs at the membrane-facing Glu residue. To verify that this concept not only holds for recombinant channel proteins in synthetic bilayers but also for their native counterparts in mitochondrial membranes, we made use of the carboxylmodifying reagent dicyclohexylcarbodiimide (DCCD). This hydrophobic compound irreversibly reacts with a number of integral membrane proteins through covalent modification of membrane-embedded Asp or Glu residues and was previously shown to modify Glu73 in VDAC1 ${ }^{48}$. Pretreatment of mitochondria with DCCD selectively abolished photolabeling of the $33 \mathrm{kDa}$ protein band with pacCer (Supplementary Fig. 7), hence providing complementary proof that the membrane-facing Glu residue in VDAC1 and VDAC2 is part of an authentic ceramide-binding site.

Loss of VDAC2 disrupts ceramide-induced apoptosis. VDAC channels are active participants in the cytosolic release of apoptogenic proteins from mitochondria. VDAC2 serves as a platform for the mitochondrial recruitment of pro-apoptotic Bcl-2 proteins Bak and $\mathrm{Bax}^{33-35}$, which can commit cells to death by permeabilizing the OMM for cytochrome $c^{17,18}$. In response to various apoptotic stimuli, VDAC1 forms oligomers and participates in the assembly of a cytochrome $c$-conducting pore ${ }^{49,50}$. Identification of a ceramide-binding site on VDAC1 and VDAC2 raised the question whether ceramides exert their apoptotic activity by interacting with these proteins. To address this, we employed an engineered ceramide transfer protein equipped with an OMM anchor, mitoCERT (Fig. 6a). We previously demonstrated that human HCT116 colon cancer cells expressing mitoCERT undergo Bax-dependent apoptosis by mistargeting newly synthesized ER ceramides to mitochondria (Fig. 6b) ${ }^{25}$. This led us to determine the impact of VDAC removal on mitoCERT-induced apoptosis in HCT116 cells. Loss of VDAC1, VDAC2, or both was verified by immunoblotting and IGF analysis of mitochondria photolabeled with pacCer (Supplementary Fig. 8). Expression of mitoCERT in wild-type HCT116 cells triggered apoptosis, as indicated by cleavage of caspase substrate PARP1 (Fig. 6c). No PARP1 cleavage was observed in cells expressing a mitoCERT variant that lacked the ceramide transfer or START domain, mitoCERT $\triangle$ START. Loss of VDAC1 had no effect on the ability of mitoCERT to induce PARP1 cleavage. In contrast, VDAC2 removal rendered cells partially resistant to mitoCERT-induced PARP1 cleavage, especially in the absence of VDAC1 (Fig. 6c). 
a
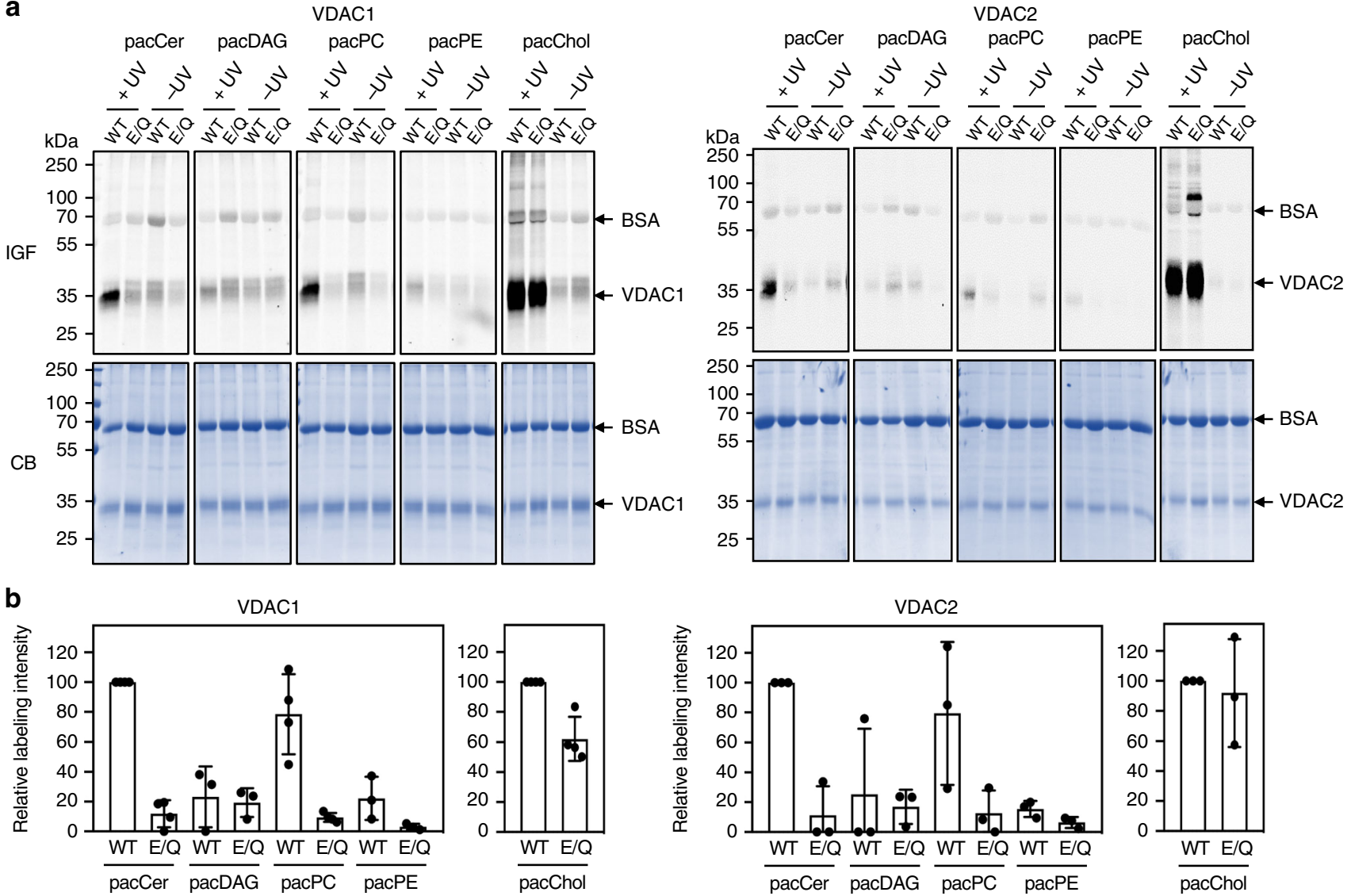

Fig. 4 The bilayer-facing Glu is a critical determinant of pacCer photolabeling of VDACs. a Human VDAC1, VDAC1 E73Q, VDAC2, and VDAC2E84Q were produced in E. coli, purified, reconstituted in liposomes, and incubated for $30 \mathrm{~min}$ at $37^{\circ} \mathrm{C}$ with liposomes containing $1 \mathrm{~mol} \%$ of pacCer or photoactive and clickable analogs of diacylglycerol (pacDAG), phosphatidylcholine (pacPC), phosphatidylethanolamine (pacPE), or cholesterol (pacChol). Samples were UV irradiated and then click-reacted with AF647- $\mathrm{N}_{3}$, subjected to SDS-PAGE, and analyzed by IGF and CB staining. b Quantitative analysis of relative labeling efficiencies of reconstituted VDAC1, VDAC1 ${ }^{\mathrm{E7} 3 \mathrm{Q}}, \mathrm{VDAC2}$, and $\mathrm{VDAC2} \mathrm{E}^{\mathrm{E} 4 \mathrm{Q}}$ with pacLipids as indicated in a. Data are means $\pm \mathrm{s}$.d.; $n \geq 3$. Source data

Glu84 in VDAC2 is critical for ceramide-induced apoptosis. We next analyzed individual wild type and ceramide-binding defective VDAC channels for their ability to support mitoCERTinduced apoptosis. To this end, HCT116 VDAC1/2 double KO cells were stably transduced with haemagglutinin (HA)-tagged VDAC1, VDAC1 ${ }^{\mathrm{E} 73 \mathrm{Q}}$, VDAC2, or VDAC2 $\mathrm{E} 84 \mathrm{Q}$ (Fig. 7a). Mitochondrial localization of the tagged channels was confirmed by immunofluorescence microscopy (Supplementary Fig. 9). Heterologous expression of VDAC2, but not VDAC1, restored mitoCERT-induced apoptosis in the double KO cells, as evidenced by PARP1 cleavage and proteolytic activation of caspase3 , the principal executioner caspase in apoptotic cells (Fig. 7a-c). This confirmed that VDAC2, unlike VDAC1, is a key player in ceramide-mediated cell death. Strikingly, replacing Glu84 with Gln in VDAC2 greatly reduced its ability to support mitoCERTinduced apoptosis in the double $\mathrm{KO}$ cells (Fig. 7a-c), indicating that cell death triggered by a rise in mitochondrial ceramides requires a ceramide-binding competent form of VDAC2. While VDAC1 or VDAC2 removal had no major impact on cellular Bax levels, cells lacking VDAC2 had strongly reduced Bak levels (Supplementary Fig. 10a), consistent with the unique role of VDAC2 in stabilizing $\mathrm{Bak}^{33}$. Reintroducing VDAC2 or VDA$\mathrm{C} 2{ }^{\mathrm{E} 84 \mathrm{Q}}$ in VDAC1/2 double $\mathrm{KO}$ cells in each case fully restored Bak levels to those in wild-type cells (Supplementary Fig. 10b), indicating that an intact ceramide-binding site is dispensable for VDAC2-mediated stabilization of Bak. This notion is supported by the finding that the Bak-stabilizing activity of VDAC2 requires isoform-specific sequence motifs located outside of the region involved in ceramide binding ${ }^{33}$.

\section{Discussion}

The current study identified a role of VDAC2 as a direct and specific effector of ceramide-mediated cell death. This function critically relies on a uniquely positioned charged Glu residue that mediates direct contact with the ceramide head group in the bilayer interior, potentially driven by electrostatic attraction. We speculate that an amide-containing backbone combined with a small polar head group renders ceramide the preferred lipid binding partner of VDAC isoforms containing the membraneburied Glu residue. Although it is energetically unfavorable for charged residues to face the bilayer's hydrophobic core, recent work revealed that the $\mathrm{pKa}$ of Glu73 in VDAC1 is closely tuned to the physiological $\mathrm{pH}$ of the cytosol $(\mathrm{pKa} \sim 7.4)^{51}$. This implies that under stress-free conditions, the membrane-buried Glu is in its deprotonated fully charged state at least a significant amount of time. Interestingly, ceramide binding to the late endosomal protein LAPTM4B depends on a membrane-embedded aspartate ${ }^{52}$. We anticipate that also other proteins with charged acidic residues in their membrane spans may have affinity for ceramide and potentially participate in ceramide-operated signaling pathways.

While ceramide-induced apoptosis requires $\mathrm{Bax}^{8,24,25}$, a recent study revealed that $\mathrm{VDAC} 2$ specifies Bax recruitment to 

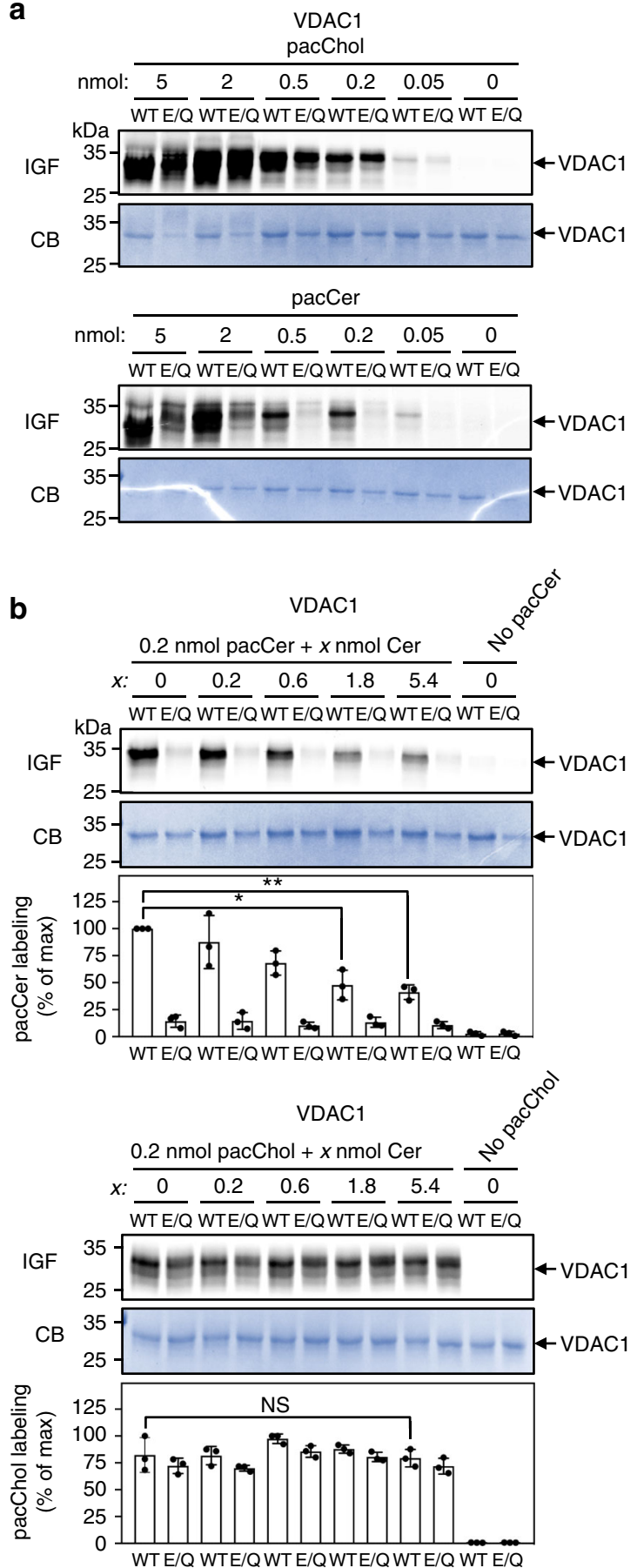
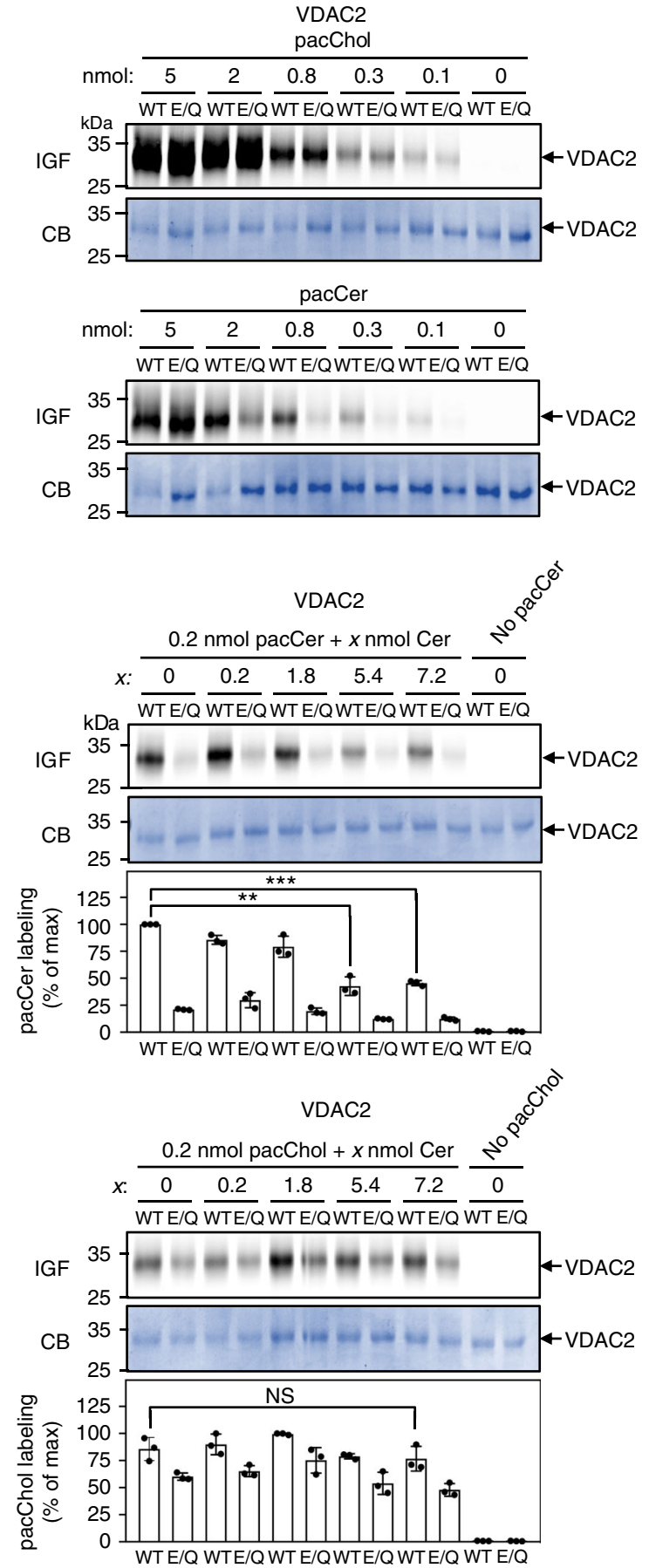

Fig. 5 Competitive inhibition of pacCer photolabeling of VDACs by $\mathrm{C}_{16}$-ceramide. a VDAC1, VDAC1 $773 \mathrm{Q}$, VDAC2, and VDAC1E84Q proteoliposomes were photolabeled with the indicated amount of pacCer or pacChol added from ethanolic stocks. Next, samples were click-reacted with $A F 647-N_{3}$, subjected to SDS-PAGE, and analyzed by IGF and CB staining. b VDAC1, VDAC1E73Q, VDAC2, and VDAC1E84Q proteoliposomes were photolabeled with $0.2 \mathrm{nmol}$ pacCer or pacChol in the presence of the indicated amounts of natural $\mathrm{C}_{16}$-ceramide (Cer) added from ethanolic stocks and then processed as in $\mathbf{a}$. Relative labeling efficiencies were quantified and expressed as $\%$ of control $(0.2 \mathrm{nmol}$ pacCer or pacChol in the absence of Cer $)$. Data are means $\pm s . d$.; $n=3$; ${ }^{\star} p<$ $0.05,{ }^{* \star} p<0.01$, and ${ }^{\star * \star} p<0.001$ by two-tailed paired $t$-test. Source data

mitochondria and concomitantly ensures Bax inhibition by mediating its retrotranslocation into the cytosol ${ }^{34}$. By establishing a dynamic equilibrium between mitochondrial and cytosolic Bax pools, this VDAC2-dependent shuttling is ideally suited for regulation by pro- and anti-apoptotic cues. Our present findings suggest that ceramide binding to VDAC2 may commit cells to death by blocking Bax retrotranslocation. This concept is distinct from previous models postulating that ceramides accumulating in the OMM: (i) self-assemble into cytochrome c-conducting channels ${ }^{27,28}$; (ii) form lipid macrodomains into which Bax inserts and functionalizes as a pore ${ }^{30,31}$; (iii) affect mitochondrial shape to facilitate Bax recruitment and apoptosis ${ }^{53}$; (iv) require metabolic conversion to gain apoptogenic activity ${ }^{32}$.

How ceramide binding tips the balance in VDAC2-mediated shuttling of Bax to trigger mitochondrial apoptosis remains to be established. Our simulations and photoaffinity experiments 

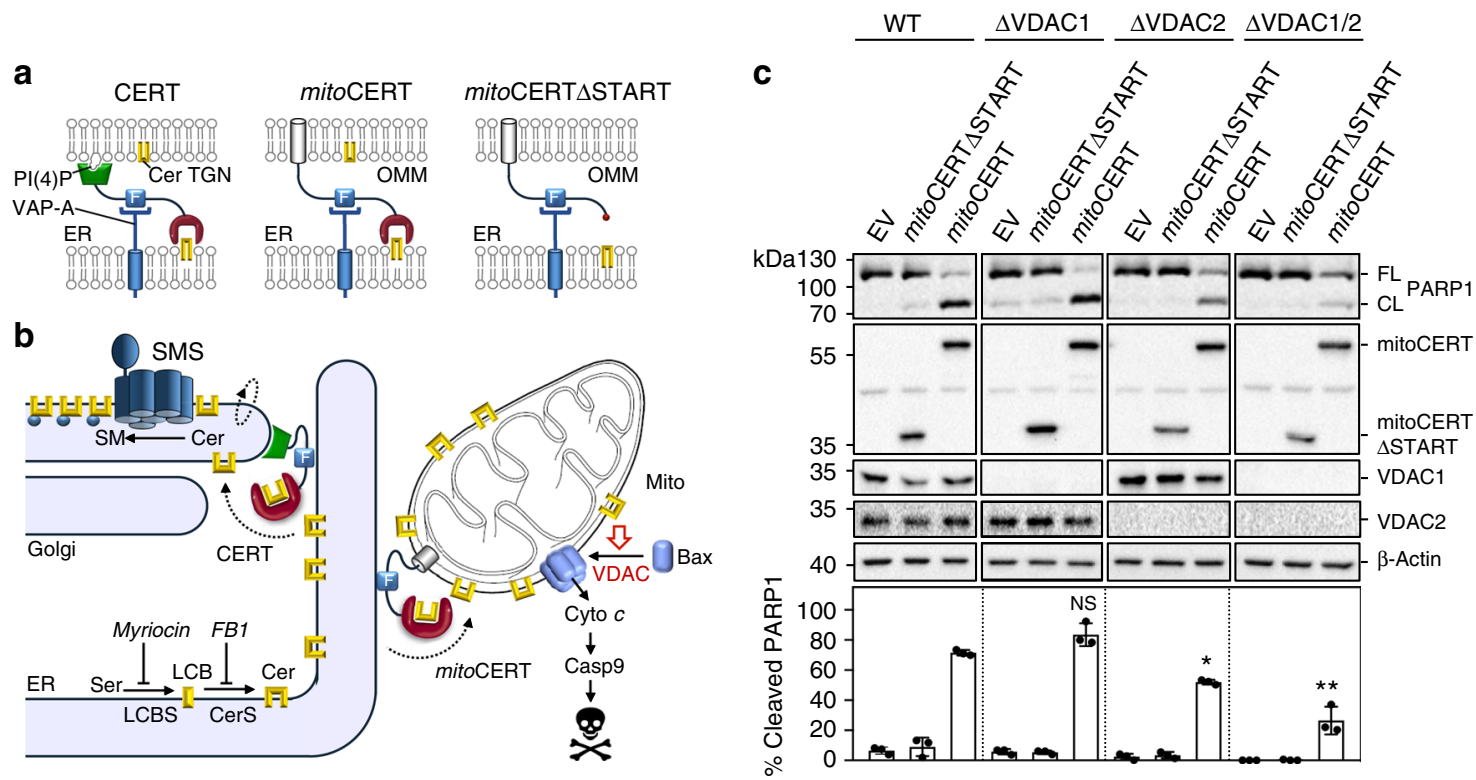

Fig. 6 VDAC2 removal disrupts ceramide-induced apoptosis. a Schematic outline of ceramide transfer protein CERT, mitoCERT, and mitoCERTASTART. MitoCERT was created by swapping the Golgi-targeting pleckstrin homology domain of CERT against the OMM anchor of AKAP1. Removal of the ceramide transfer or START domain yielded mitoCERT $\triangle$ START. All three proteins bind the ER-resident protein VAP-A via their FFAT motif (F). Cer ceramide, PI(4)P phosphatidylinositol-4-phosphate, TGN trans-Golgi network. b Ceramides (Cer) are synthesized through N-acylation of long chain bases (LCB) by ceramide synthases (CerS) on the cytosolic surface of the ER and require CERT-mediated transfer to the Golgi for metabolic conversion into sphingomyelin (SM) by a Golgi-resident SM synthase (SMS). Expression of mitoCERT causes a diversion of this biosynthetic ceramide flow to mitochondria, triggering Bax-dependent apoptosis ${ }^{11}$. c Wild type (WT), VDAC1-KO ( $\triangle$ VDAC1), VDAC2-KO ( $\triangle$ VDAC1), and VDAC1/2 double KO ( $\triangle$ VDAC1/2) human colon cancer HCT116 cells were transfected with empty vector (EV), Flag-tagged mitoCERT, or Flag-tagged mitoCERT $\triangle S T A R T$. At $24 \mathrm{~h}$ post transfection, cells were processed for immunoblotting with antibodies against PARP1, the Flag-epitope, VDAC1, VDAC2, and $\beta$-actin. The percentage of PARP1 cleavage was quantified. Data are means \pm s.d.; $n=3 ;{ }^{\star} p<0.05$ and ${ }^{\star \star} p<0.01$ by two-tailed paired $t$-test. Source data

indicate that ceramide binding to VDACs is $\mathrm{pH}$ sensitive and controlled by the protonation state of the membrane-buried Glu. Interestingly, acidification has been shown to promote association of two VDAC1 monomers into a dimer ${ }^{51}$. Assembly of a highaffinity dimer relies on protonation of the membrane-facing Glu (E73) and likely involves hydrogen bonding between this residue and a serine (Ser43) at the dimer interface ${ }^{51}$. VDAC oligomerization has been implicated as a component of the mitochondrial pathway of apoptosis 49,50 and may be part of the mechanism by which VDAC2 stabilizes the mitochondrial pool of Bax in response to apoptotic stimulation ${ }^{34}$. In view of our present findings, a prospect that merits further investigation is whether binding of ceramide to the charged Glu residue lowers the threshold for VDAC oligomerization at neutral $\mathrm{pH}$. Ceramide binding to VDACs may also influence interactions with other proteins, as the membrane-facing Glu residue is critical for association of VDAC1 with hexokinase $\mathrm{I}^{54,55}$. VDAC-bound hexokinases are thought to play a pivotal role in promoting cell growth and survival in rapidly growing, hyperglycolytic tumors $^{56,57}$. Consequently, our current study establishes a molecular framework to unravel how ceramides execute their tumor suppressor functions.

\section{Methods}

Reagents. 1,2-Dioleoyl-sn-glycero-3-phosphocholine (DOPC), 1,2-dioleoyl-snglycero-3-phosphoethanol-amine (DOPE), and L-a-phosphatidylcholine from chicken egg (egg PC), 1-palmitoyl-2-\{12-[(7-nitro-2-1,3-benzoxadiazol-4-yl) amino]dodecanoyl $\}$-sn-glycero-3-phosphocholine (NBD-PC) and $\mathrm{C}_{16}$-ceramide (d18:1/16:0) were purchased from Avanti Polar Lipids. Alexa Fluor 647- $\mathrm{N}_{3}$ $\left(\right.$ AF647- $\mathrm{N}_{3}$ ) and Biotin- $\mathrm{N}_{3}$ were from Thermo Fischer Scientific, and TAMRABiotin- $\mathrm{N}_{3}$ from Click Chemistry Tools. The photoactive and clickable trans-sterol probe (pacChol) was from Sigma-Aldrich. A 15 carbon-long fatty acid containing a photoactivatable diazerine and clickable alkyne group, pacFA, was synthesized in three steps from commercially available educts ${ }^{36}$. Next, pacFA was coupled to D- erythro-sphingosine (Enzo Biochem) using a combination of 1-ethyl-3-(3-dimethylaminopropyl)carbodiimide (EDCI) and hydroxybenzotriazole (HOBT) as condensing reagents, yielding the photoactivatable and clickable $\mathrm{C} 15$-ceramide analog, pacCer (85\% overall yield). pacPC was synthesized starting from 1-oleoyl2-hydroxy-sn-glycero-3-phosphocholine (Avanti Polar Lipids) and pacFA under the action of $\mathrm{N}, \mathrm{N}$-dicyclohexylcarbodiimide (DCC) and 4-dimethylaminopyridine (DMAP) with satisfactory yield (39\%). pacDAG was synthesized in three steps starting from 1-oleoyl-sn-glycerol (Santa Cruz Biotechnology). First, the primary HO-group was protected with the triphenylmethyl protecting group (trityl-chloride/pyridine; $92 \%$ overall yield). The 1-acyl-3-trityloxy-glycerol obtained was coupled with the pacFA using EDCI/DMAP activation (58\% overall yield). The final deprotection step was achieved using trifluoroacetic acid (TFAA) to generate pacDAG (28\% overall yield). pacPE was synthesized in three steps starting from 1 oleoyl-2-hydroxy-sn-glycero-3-phosphoethanolamine (Avanti Polar Lipids). First, the amino-group was protected with the tert-butoxycarbonyl protecting group (ditert-butyldicarbonate/triethylamine; $98 \%$ overall yield). The N-protected lyso-PE obtained was coupled with pacFA using EDCI/DMAP activation in a good yield (52\%). The final deprotection step was achieved with TFAA to generate pacPE (35\%, overall yield).

Antibodies. Antibodies used were mouse monoclonal anti- $\beta$-actin (Sigma-Aldrich, A1978; IB 1:50,000), rabbit polyclonal anti-FLAG (Cell Signaling, 2368; IB 1:1,000), mouse monoclonal anti-mitochondrial surface protein p60 (Millipore, MAB1273; IB 1:1,000), rabbit polyclonal anti-VDAC1 (Cell Signaling, 4661; IB 1:1,000), goat polyclonal anti-VDAC2 (Abcam, Ab37985; IB 1:1,000), rabbit polyclonal antiVDAC3 (Abcam, Ab80452; IB 1:1,000), mouse monoclonal anti-TOM20 (Millipore, Mabt166; IF 1:200), rabbit polyclonal anti-TOM40 (Abcam, Ab185543; IB 1:1,000), rabbit polyclonal anti-HA (Invitrogen, 715500; IB 1:1,000; IF 1:200), rat monoclonal anti-HA (Roche, 12158167001; IB 1:1,000), rabbit monoclonal antiBax (Cell Signaling, 5023; IB 1:1,000), rabbit polyclonal anti-cleaved caspase-3 (Cell Signaling, 96611; IB 1:1,000), mouse monoclonal anti-PARP-1 (Santa Cruz, sc8007; IB 1:1,000) and rabbit polyclonal anti-calnexin (Santa Cruz, sc11397; IB 1:1,000). Goat anti-mouse (31430; IB 1:5,000), goat anti-rabbit (31460; IB 1:5,000) and donkey anti-goat IgG conjugated to horseradish peroxidase (pa1-28664; IB 1:5,000) were from Thermo Fischer Scientific. $\mathrm{Cy}^{\mathrm{Tm}}$-dye-conjugated donkey anti-mouse and donkey anti-rabbit antibodies (715-225-150, 715-225-152, 715-165-150, 715-165152, 715-175-150 and 715-175-152; IF 1:250 each) were from Jackson ImmunoResearch Laboratories. 
a

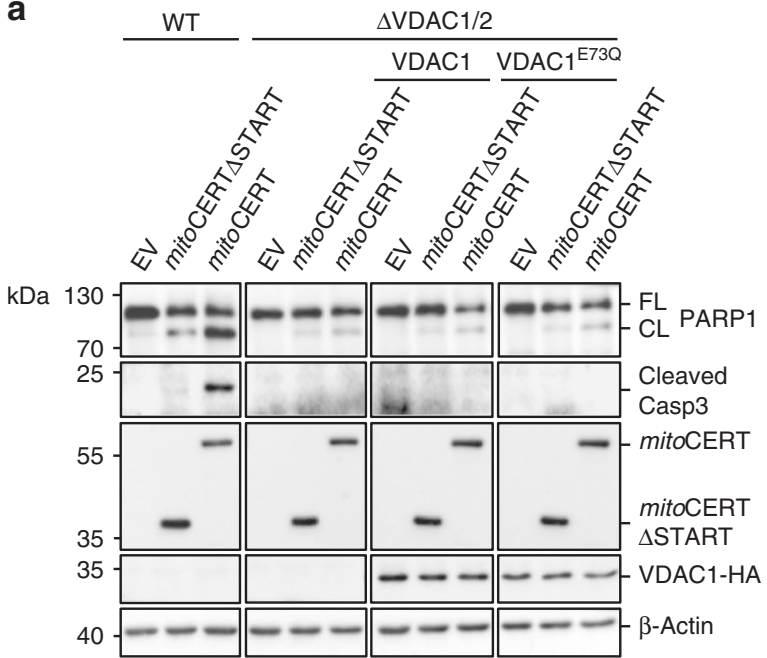

b

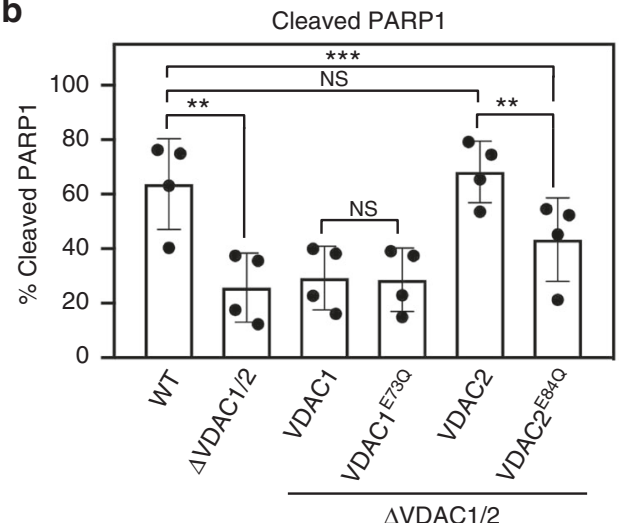

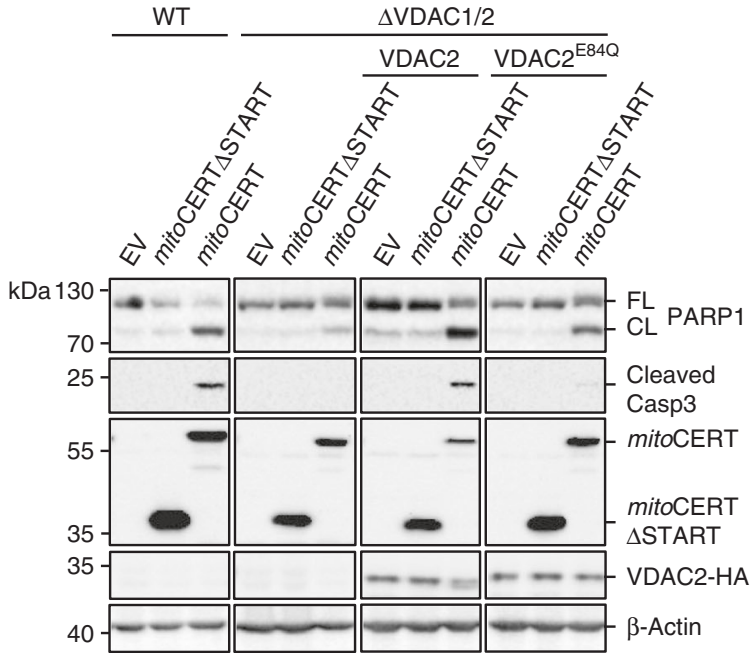

C

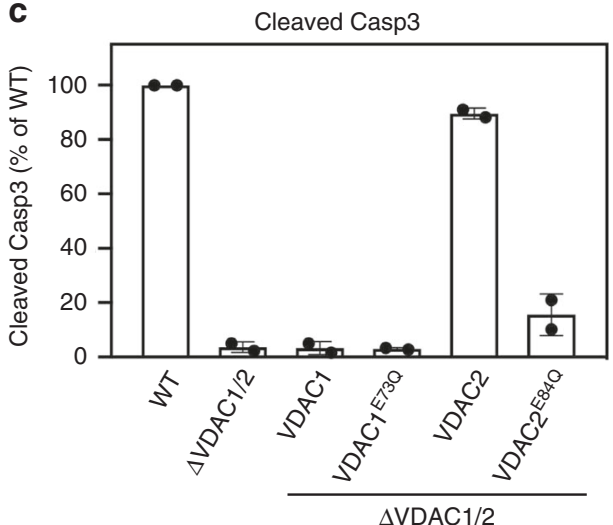

Fig. 7 Ceramide-induced apoptosis critically relies on Glu84 in VDAC2. a WT and $\triangle$ VDAC1/2 HCT116 cells stably transduced with HA-tagged VDAC1, VDAC1773Q, VDAC2, or VDAC2E84Q were transfected with empty vector (EV), Flag-tagged mitoCERT, or Flag-tagged mitoCERT $\triangle S T A R T$. At $24 \mathrm{~h}$ post transfection, cells were processed for immunoblotting with antibodies against PARP1, cleaved caspase-3 (Casp3), the Flag-epitope, the HA-epitope, and $\beta$ actin. FL full-length, $\mathrm{CL}$ cleaved. $\mathbf{b}$ Quantitative analysis of PARP1 cleavage in cells treated as in $\mathbf{a}$. Data are means \pm s.d.; $n=4 ;{ }^{*} p<0.05,{ }^{\star *} p<0.01$ and ${ }^{* \star *} p<0.001$ by two-tailed paired $t$-test. c Quantitative analysis of cleaved Casp3 in cells treated as in a. Data are means \pm s.e.; $n=2$. Source data

DNA constructs. For expression of human VDACs in E. coli, the corresponding cDNAs were PCR amplified using Phusion high-fidelity DNA polymerase (Thermo Fischer Scientific) and inserted via NdeI and XbaI (VDAC1) or XhoI and XbaI sites (VDAC2) into bacterial expression vector pCold I (Takara Bio, USA). For retroviral transduction studies, DNA fragments encoding human VDACs with a $C$ terminal HA tag (YPYDVPDYA) were created by PCR and inserted via NotI and XhoI sites into lentiviral expression vector pLNCX2 (Takara Bio, USA). Single amino acid substitutions were introduced using the QuikChange II site-directed mutagenesis method (Alignet, USA). Primers used for cloning and site-directed mutagenesis are listed in Supplementary Table 3. Mammalian expression constructs encoding FLAG-tagged mitoCERT and mitoCERT $\triangle$ START were described previously ${ }^{25}$. All expression constructs were verified by DNA sequencing.

Cell culture and transfection and RNAi. Human cervical carcinoma HeLa cells (ATCC CCL-2) were cultured in Dulbecco's modified Eagle's medium (DMEM) supplemented with $4.5 \mathrm{~g} / \mathrm{l}$ glucose, $2 \mathrm{mM}$ L-glutamine and $10 \% \mathrm{FBS}$. Human colon carcinoma HCT116 cells (ATCC CCL-247) were cultured in McCoy's medium supplemented with 10\% FBS. Human embryonic kidney HEK293T cells (ATCC CRL-3216) were cultured in DMEM supplemented with 10\% FBS. Cells were transfected with DNA constructs using Effectene (Qiagen) according to the manufacturer's instructions unless stated otherwise. Treatment of HeLa cells with siRNA (Qiagen) was performed using Oligofectamine reagent (Invitrogen) according to the manufacturer's instructions. siRNA target sequences were: NS (nonsense), 5'-AAUUCUCCGAACGUGUCACGU-3'; VDAC1, 5'-ACACUA GGCACCGAGAUUAUU-'3; VDAC2, 5'-AAUACAAGUGGUGUGAGUAUU-3'.

Generation of VDAC KO cell lines. To knock out VDAC1 and VDAC2 in HCT116 cells, we obtained a mix of three different CRISPR/Cas9 plasmids per gene and the corresponding HDR plasmids from Santa Cruz (sc-418200, sc-416966). The VDAC1-specific gRNA sequences were: A/sense, 5'-TTGAAGGAATTTACA AGCTC-3'; B/sense, 5'-CGAATCCATGTCGCAGCCC-3'; C/sense, 5'-CTTACA CATTAGTGTGAAGC-3'. The VDAC2-specific gRNA sequences were: A/sense, 5'-AGAAATCGCAATTGAAGACC-3'; B/sense, 5'-GCCCTTAAGCAGCACAG CAT-3'; C/sense, 5'-TAATGTGACTCTCAAGTCCT-3'. HCT116 cells were transfected with both plamid mixes and grown for $48 \mathrm{~h}$ without selection. Next, cells were grown for 2 weeks under selective pressure with $2 \mu \mathrm{g} / \mathrm{ml}$ puromycin. Individual drug-resistant clones were picked and analyzed for VDAC1 and VDAC2 expression by immunoblot analysis. A VDAC1/2 double $\mathrm{KO}$ cell line was generated from $\triangle \mathrm{VDAC1}$ cells as described above following ejection of the puromycin selectable marker using Cre vector (Santa Cruz, sc-418923) according to the manufacturer's instructions.

Retroviral transduction. HCT116 VDAC1/2 double KO cells stably expressing HA-tagged $\mathrm{VDAC1}, \mathrm{VDAC1}{ }^{\mathrm{E} 73 \mathrm{Q}}$, VDAC2, or $\mathrm{VDAC2} \mathrm{E}^{\mathrm{E} 4 \mathrm{Q}}$ were created by retroviral transduction. To this end, HEK293T cells were co-transfected with pLNX2VDAC-HA expression constructs and packaging vectors (Clontech) using Lipofectamine 3000 (Invitrogen) according to the manufacturer's instructions. The culture medium was changed $6 \mathrm{~h}$ post transfection. After $48 \mathrm{~h}$, the retroviruscontaining medium was harvested, filtered through a $0.45 \mu \mathrm{m}$ filter, mixed 1:1 (v/v) with McCoy's growth medium, supplemented with $8 \mu \mathrm{g} / \mathrm{ml}$ polybrene, and used to transduce HCT116 VDAC1/2 double KO cells. Hygromycin $(300 \mu \mathrm{g} /$ $\mathrm{ml}$ ) was added $6 \mathrm{~h}$ post-infection and selective medium was exchanged daily. After 3-5 days, positively transduced cells were selected and analyzed for expression of HA-tagged VDACs by immunoblot analysis and immunofluorescence microscopy. 
Reconstitution of recombinant VDACs. VDAC-encoding pCold I constructs were transformed in E. coli BL21 (DE3) $\Delta$ Omp9 cells (a kind gift from Dr. LarsOliver Essen, Philips-Universität Marburg, Germany). Transformants were grown at $37^{\circ} \mathrm{C}$ to early exponential phase in $\mathrm{LB}$ medium containing $100 \mu \mathrm{g} / \mathrm{ml}$ ampicillin and cooled for $30 \mathrm{~min}$ at $4{ }^{\circ} \mathrm{C}$ prior to addition of $1 \mathrm{mM}$ IPTG. Growth was continued for $24 \mathrm{~h}$ at $15^{\circ} \mathrm{C}$. Cells were collected by centrifugation and lysed in TEN buffer $(50 \mathrm{mM}$ Tris/ $\mathrm{HCl} \mathrm{pH} \mathrm{8.0,100} \mathrm{mM} \mathrm{NaCl})$ supplemented with $2.5 \%$ Triton X100 and protease inhibitor cocktail (PIC; $1 \mu \mathrm{g} / \mathrm{ml}$ apoprotinin, $1 \mu \mathrm{g} / \mathrm{ml}$ leupeptin, 1 $\mu \mathrm{g} / \mathrm{ml}$ pepstatin, $5 \mu \mathrm{g} / \mathrm{ml}$ antipain, $157 \mu \mathrm{g} / \mathrm{ml}$ benzamide) through micro-tip sonication. Inclusion bodies were collected by centrifugation $\left(30 \mathrm{~min}, 2.500 \times g, 4^{\circ} \mathrm{C}\right)$, washed three times in TEN buffer with, and then three times in TEN buffer without, Triton $\mathrm{X}-100$. Inclusion bodies were diluted 1:10 by dropwise addition into $25 \mathrm{mM} \mathrm{Na}^{+} \mathrm{PO}_{4} \mathrm{pH} 7.0,100 \mathrm{mM} \mathrm{NaCl}, 6 \mathrm{M}$ guanidine hydrochloride, $1 \mathrm{mM}$ EDTA and $10 \mathrm{mM}$ DTT while stirring, and stirred overnight at $4{ }^{\circ} \mathrm{C}$. Next, the suspension was diluted 1:10 by dropwise addition to $25 \mathrm{mM} \mathrm{Na}^{+} \mathrm{PO}_{4} \mathrm{pH} 7.0,100$ $\mathrm{mM} \mathrm{NaCl}, 1 \mathrm{mM} \mathrm{EDTA}$, and 2.2\% lauryldimethylamine oxide (LDAO), and stirred overnight at $4{ }^{\circ} \mathrm{C}$. Finally, the suspension was diluted 1:10 by dropwise addition to $25 \mathrm{mM} \mathrm{Na}{ }^{+} \mathrm{PO}_{4} \mathrm{pH} 7.0,10 \mathrm{mM} \mathrm{NaCl}, 1 \mathrm{mM}$ EDTA, $0.1 \% \mathrm{LDAO}$, and $1 \mathrm{mM}$ DTT, stirred overnight at $4{ }^{\circ} \mathrm{C}$, and loaded on a Fractogel EMD-SE Hicap cationexchange column (Merck Millipore). VDAC proteins were eluted with a linear $\mathrm{NaCl}$ concentration gradient in $25 \mathrm{mM} \mathrm{Na}^{+} \mathrm{PO}_{4} \mathrm{pH} 7.0,10 \mathrm{mM} \mathrm{NaCl}, 1 \mathrm{mM}$ EDTA, $1 \mathrm{mM}$ DTT, and $0.1 \%$ LDAO on a ÄKTAprime plus protein purification system (GE Healthcare Life Sciences). Peak fractions were pooled, concentrated on an Amicon Ultra-4 unit (MWCO $10 \mathrm{kDa}$; Merck Millipore), and loaded on a Superose 12 10/300 GL size exclusion column (GE Healthcare Life Sciences). Elution was in $10 \mathrm{mM}$ Tris/HCl, pH 7.0, $100 \mathrm{mM} \mathrm{NaCl}$, and $0.05 \%$ LDAO. Purified VDAC proteins in peak fractions were pooled, quantified using Amido Black ${ }^{58}$, and then reconstituted at a concentration of $1 \mathrm{mg} / \mathrm{ml}$ in egg PC vesicles supplemented with $1 \mathrm{~mol} \% \mathrm{NBD}-\mathrm{PC}$ at a protein to lipid ratio of 1:300 (mol/mol). To this end, egg PC and NBD-PC dissolved in $\mathrm{CH}_{3} \mathrm{Cl}$ were dried and dissolved in buffer $\mathrm{R}$ $(100 \mathrm{mM} \mathrm{KCl}, 10 \mathrm{mM}$ MOPS/Tris $\mathrm{pH} 7.0)$ by vortexing and sonication. LDAO was added in 10-fold molar excess over lipids. The concentration of LDAO in the protein sample was adjusted to that in the lipid sample. Both samples were incubated separately at RT for $20 \mathrm{~min}$, mixed at a 1:1 (v/v) ratio, and incubated again for $30 \mathrm{~min}$. SM2 Biobeads (Bio-Rad Laboratories) pretreated according to the manufacturer's instructions were added to the lipid-protein mixture in 30-fold excess over detergent $(\mathrm{w} / \mathrm{w})$. After incubation overnight at $4{ }^{\circ} \mathrm{C}$ on a rotating wheel, the beads were removed by centrifugation and the proteoliposome-containing supernatant was aliquoted, snap-frozen in liquid $\mathrm{N}_{2}$, and stored at $-80^{\circ} \mathrm{C}$. To check reconstitution efficiency, an aliquot of the proteoliposomes was subjected to density flotation analysis. To this end, proteoliposomes were mixed 1:1 (v/v) with 80\% Accudenz (Accurate Chemical \& Scientific Corporation, USA) in buffer R, transferred to the bottom of a $5 \mathrm{ml}$ ultracentrifuge tube, and overlayed with $30 \%$, $20 \%, 10 \%$, and $0 \%$ Accudenz prepared in reconstitution buffer. After centrifugation for $1 \mathrm{~h}$ at $100,000 \times g$ at $4{ }^{\circ} \mathrm{C}, 10 \times 0.5 \mathrm{ml}$ fractions were collected from top to bottom. Fractionation profiles of NBD-PC and VDACs were determined by TLC analysis and SDS-PAGE followed by Coomassie blue staining, respectively.

Photoaffinity labeling of recombinant VDACs. Liposomes used for photoaffinity labeling of reconstituted VDACs were prepared from a defined lipid mixture (DOPC/DOPE/pacLipid, 80/20/1 mol\%) in $\mathrm{CHCl}_{3} /$ methanol (9/1, v/v). In brief, 10 $\mu \mathrm{mol}$ of total lipid was dried in a Rotavap and the resulting lipid film was resuspended in $1 \mathrm{ml}$ buffer $\mathrm{L}(50 \mathrm{mM}$ Tris- $\mathrm{HCl}, \mathrm{pH} 7.4,50 \mathrm{mM} \mathrm{NaCl}$ ) by vigorous vortexing and sonication, yielding a $10 \mathrm{mM}$ lipid suspension. Liposomes with an average diameter of $\sim 100 \mathrm{~nm}$ were obtained by sequential extrusion of the lipid suspension through $0.4,0.2$, and $0.1 \mu \mathrm{m}$ track-etched polycarbonate membranes (Whatman-Nucleopore) using a mini-extruder (Avanti Polar Lipids). VDAC proteoliposomes were diluted in buffer $\mathrm{R}$ to a final protein concentration of $0.1 \mathrm{mg} /$ $\mathrm{ml}$, mixed with an equal volume of $10 \mathrm{mM}$ liposomes containing $1 \mathrm{~mol} \%$ pacLipid, and incubated for $30 \mathrm{~min}$ at $37^{\circ} \mathrm{C}$ with gentle shaking. For the experiments shown in Figs. 3 and 4, VDAC proteoliposomes were incubated with pacLipids added from ethanolic stocks $(0.2 \mathrm{nmol}$ in $100 \mu \mathrm{l}$ reaction volume, unless indicated otherwise). To determine the impact of $\mathrm{pH}$ on pacCer labeling of VDAC1, VDAC1 proteoliposomes were collected by high-speed centrifugation and then resuspended in the following buffers prior to photolabeling: (a) $100 \mathrm{mKCl}, 50 \mathrm{mM}$ MES-NaOH pH 5.0; (b) $100 \mathrm{mKCl}, 50 \mathrm{mM}$ MES-NaOH pH 6.0; (c) $100 \mathrm{mKCl}, 10 \mathrm{mM}$ MOPS/ Tris $\mathrm{pH} 7.0$; (d) $100 \mathrm{mKCl}, 50 \mathrm{mM}$ HEPES $\mathrm{pH}$ 8.0. The mixtures were placed on ice and irradiated for $90 \mathrm{~s}$ using a $1000 \mathrm{~W}$ mercury lamp equipped with a dichroic mirror and a $345 \mathrm{~nm}$ bandpass filter (Newport) at $30 \mathrm{~cm}$ distance. Proteins were recovered by chloroform-methanol precipitation after external addition of BSA as a carrier and the air-dried protein pellet was dissolved in 1\% SDS in PBS with vigorous shaking for $10 \mathrm{~min}$ at $70^{\circ} \mathrm{C}$. Samples were click-reacted with $80 \mu \mathrm{M}$ AF647- $\mathrm{N}_{3}$ in $1 \mathrm{mM}$ TCEP (Tris(2-carboxyethyl)phosphine hydrochloride), $0.1 \mathrm{mM}$ TBTA (Tris[(1-benzyl-1H-1,2,3-triazol-4-yl)methyl] amine), and $1 \mathrm{mM} \mathrm{CuSO}_{4}$ for $1 \mathrm{~h}$ at $37^{\circ} \mathrm{C}$. After addition of $0.25 \mathrm{vol}$ of $5 \times$ Sample buffer $(0.3 \mathrm{M}$ Tris/ $\mathrm{HCl}, \mathrm{pH} 6.8$, $10 \%$ SDS, $50 \%$ glycerol, $0.025 \%$ bromphenol blue, and $10 \% \beta$-mercaptoethanol), samples were boiled for $5 \mathrm{~min}$ at $95^{\circ} \mathrm{C}$, subjected to SDS-PAGE and analyzed by IGF using a Typhoon FLA 9500 (GE Healthcare) with a $635 \mathrm{~nm}$ laser and LPR filter. Fluorescence intensities were quantified using ImageQuant TL software. Next, gels were stained with Coomassie blue and the amount of recombinant protein was determined by measuring the staining intensity using Image Lab 5.2 software (Bio-Rad Laboratories). Fluorescence intensities of recombinant protein were corrected for background fluorescence in the same lane and then divided by the total amount of protein. The specific fluorescence intensity of UV-irradiated protein was determined after subtraction of fluorescence intensity of non-UVirradiated protein.

Identification of pacCer-photolabeled proteins. Post-nuclear supernatants, cytosol, total membranes, and membrane fractions enriched in mitochondria or ER were prepared from HeLa cells ${ }^{25}$. Subcellular fractions were diluted in buffer R $(10 \mathrm{mM}$ Tris, pH 7.4, 0.25 M sucrose) supplemented with $0.1 \mathrm{mM}$ PMSF and PIC to $0.45-0.70$ $\mathrm{mg} / \mathrm{ml}$ total protein and then mixed with an equal volume of $10 \mathrm{mM}$ liposomes containing $1 \mathrm{~mol} \%$ pacCer prepared in buffer R as above. Samples were incubated for $60 \mathrm{~min}$ at $37^{\circ} \mathrm{C}$ with gentle shaking, placed on ice, and then UV irradiated for $90 \mathrm{~s}$ Proteins were recovered by chloroform-methanol precipitation, click reacted with AF647- $\mathrm{N}_{3}$ and then subjected to IGF as above. For identification of the mitochondriaassociated 33kDa-protein band, an HeLa cell-derived membrane fraction enriched in mitochondria $(20 \mu \mathrm{g}$ total protein) was photolabeled with pacCer as above. Experiments in which pacCer was omitted from the liposomes served as controls. Proteins were recovered by chloroform-methanol precipitation, dissolved in $50 \mu \mathrm{l}$ 1\% SDS PBS by vigorous vortexing and sonication in a water bath. After addition of $50 \mu \mathrm{l}$ PBS, the sample was click-reacted with $1 \mathrm{mM}$ TAMRA-Biotin- $\mathrm{N}_{3}$ as above. Next, two-third of the sample was mixed with $100 \mu$ slurry of NeutrAvidin ${ }^{\text {nx }}$ Agarose Resin (Thermo Fisher Scientific, USA) pre-equilibrated in PBS/0.5\% SDS and incubated for $2 \mathrm{~h}$ at RT while shaking. The beads were washed five times in $0.6 \mathrm{ml}$ PBS $/ 0.5 \%$ SDS and then incubated in Sample buffer for $5 \mathrm{~min}$ at $95^{\circ} \mathrm{C}$ to elute bound proteins. Proteins in total input, flow-through, wash, and eluate were recovered by chloroform-methanol precipitation and processed for IGF analysis as above. The pacCer-labeled $33-\mathrm{kDa}-$ protein band in the eluate fraction was excised from the gel and digested by trypsin (Promega, V5111) for $6 \mathrm{~h}$ at $37^{\circ} \mathrm{C}$. Protein fragments were collected, concentrated, and analyzed by LC-MS/MS in an amaZon Spped ETD ion Trap mass spectrometer (Bruker, USA) connected to an UltiMate 3000 nano LC system (Thermo Fisher Scientific). Peptides were separated on a C18 column using a two-buffer system with water/acetonitrile/formic acid (99/1/0.1, v/v/v) and water/acetonitrile/formic acid (20/ 80/0.1, v/v/v). Raw MS data were converted to peak lists using Data Analyzer 4.1 (Bruker, USA) and noise filtered using an in-house developed script. The spectra were searched with Mascot (precursor mass tolerance 0.8 Da; product mass tolerance 0.4 Da; 2 missed cleavages) against all human proteins in the Swissprot (v56.2) database. Peptide identifications were accepted with a score greater than 20 and a $p$-value smaller than 0.01 , and proteins were identified with at least two unique peptides.

MD simulations. For VDAC MD simulations, a well-resolved structure of mouse VDAC1 (PDB: 4 C69 at $2.8 \AA$ resolution) ${ }^{41}$ was selected as a main template. This structure shares a $\beta$-barrel backbone RMSD of $1.7 \AA$ with a structure of VDAC2 from zebrafish (PDB: 4BUM) ${ }^{42}$. Based on the assumption of identical secondary structures, VDAC1 side chains were mutated to the mouse sequences of VDAC2 (NCBI ID:NP_035825.1) and VDAC3 (NCBI ID:NP_035826.1) to obtain structures of all three isoforms for comparison using the PyMOL software. The mutation process was semi-automated and leveraged the used CG representation (see below), in which side chains are represented by up to four particles and are therefore simple to replace. The $N$-terminal $\alpha$-helix of the VDAC2 was truncated to match VDAC1 sequence length (with no impact to lipid interactions because the helix sits inside the channel's $\beta$-barrel). VDACs were embedded in an outer mitochondrial membrane mimic with about 630 lipids using the insane script ${ }^{59}$. Membrane composition was based on Horvath and Daum ${ }^{43}$, with a mixture of POPC/POPE/POPI/cholesterol $(52 / 14 / 19 / 15, \mathrm{~mol} \%)$ in the inner leaflet and POPC/POPE/POPI/cholesterol (42.5/32/5/15.5, mol\%) supplemented with $5 \mathrm{~mol} \%$ $\mathrm{C}_{16}$-ceramide in the outer leaflet. About $150 \mathrm{mM} \mathrm{NaCl}$ was added to the system, plus an excess of $\mathrm{Na}^{+}$ions to reach charge neutrality. The Martini coarse-grain (CG) force field ${ }^{60}$ was used to model the simulated systems, together with the ElNeDyn elastic-network approach to restrain the protein secondary structure ${ }^{61}$. Simulations were run with GROMACS version $5.0^{62}$ in the isothermal-isobaric $(\mathrm{NpT})$ ensemble, at $300 \mathrm{~K}$ and 1 bar. Temperature was controlled using the $\mathrm{v}$ rescale thermostat with a coupling constant of 1.0 ps. Pressure was coupled semiisotropically, independently in $x y$ and $z$, using the Parrinello-Rahman barostat with a coupling constant of 12.0 ps and a compressibility of $4.5 \times 10^{-5} \mathrm{bar}^{-1}$. Standard Martini parameters were used to represent interparticle interactions ${ }^{60,63}$. electrostatic interactions were modeled with a Coulombic potential cutoff at 1.2 $\mathrm{nm}$, shifted to zero along the entire range, in conjunction with an implicit screening constant of 15 . The Van der Waals interactions were calculated using a shifted Lennard-Jones potential, with a cutoff of $1.2 \mathrm{~nm}$ and a shift to zero from $0.9 \mathrm{~nm}$. A simulation integration time step of $20 \mathrm{fs}$ was used, and particle neighborsearching was performed over a $1.4 \mathrm{~nm}$ radius once every 10 simulation steps. As the apparent $\mathrm{pKa}$ of the membrane-facing glutamate E73 in VDAC1 is closely tuned to the physiological $\mathrm{pH}$ of the cytosol $(\mathrm{pKa} \sim 7.4)^{51}$, main simulations of VDAC1 and VDAC2 were initially done with deprotonated E73/E84 in six replicates $(0.3 \mathrm{~ms}$ total $)$ each. Other simulated systems include VDACs with protonated E73/E84, or with E73Q/E84Q or E73D/E84D mutations. VDAC1 with deprotonated E73 was also simulated in 100\% DMPC. The total simulation time for all 
systems was $1.23 \mathrm{~ms}$ (Supplementary Table 2). Trajectories were saved and analyzed every 600 ps.

Simulation analysis. Two main types of analysis were performed: contact analysis and space occupation analysis. Contact analyses were performed either as residuediscriminated analyses or as residence-time distributions. Residue-discriminated contacts were calculated by finding all frames of ceramide (particles AM1 or AM2) or cholesterol (particle ROH) head groups within $7 \AA$ of any relevant residue particle. To allow comparison between simulations of different lengths, the number of in-contact frames was normalized by the total number of frames. Contact residence-time distributions were plotted by logarithmically histogramming the duration of all individual continuous binding events of each single ligand to the VDAC-binding site. To correctly convey the impact of long-term binding events, $y$ values indicate the sum of binding durations in that bin. To compare between simulations of different duration, the $y$-values were further normalized for the respective total simulation time. Analysis of continuous contact times is very sensitive to the use of a sharp cutoff, and long binding events will be underestimated if the ligand briefly drifts past the threshold, even if not actually leaving the binding site. To correct for this, for binding duration analysis a contact was defined when a head group particle (AM1 or AM2 in ceramide; PO4 or NC3 in DMPC) came within $8 \AA$ of at least two groups of residues, out of three defined on the $\beta$-strands that compose the binding site (VDAC1 residues 58-60, 73-75, and 81-83). Spuriously brief contacts, or spuriously brief losses of contact, were further filtered by applying a smoothing window five-frames wide; if there were contacts on three or more of those frames the center frame was set to be in-contact, otherwise it was set to be not-in-contact. Binding events that were still bound at the end of a simulation run were disregarded. Space occupation analyses were performed by gridding the simulation box at $5 \AA$ spacing and comparing ceramide versus cholesterol occupancy. The number of occupied frames for each cell was calculated for the entire simulation time, and then normalized by the total number of frames, yielding a grid of occupancy values ranging from zero (never present in that cell) to one (always present). Occupancy volumes were drawn as isooccupancy surfaces at given thresholds ( $10 \%$ for ceramide, $20 \%$ for cholesterol), adjusted to compensate for the difference in the number of lipid molecules. To allow meaningful occupancy surfaces to be drawn relative to the protein, its position and rotation in the $x y$ plane were aligned beforehand across all analyzed trajectories.

Algorithms and statistical analysis. Analysis algorithms were programmed in Python, using MDAnalysis (https://www.mdanalysis.org) ${ }^{64,65}$ and NumPy ${ }^{66}$ packages. The MDreader package (https://github.com/mnmelo/MDreader) was used to allow analysis parallelization, which was essential in tackling the multigigabyte trajectory analysis in a short time. Two types of statistical analyses were performed on the distribution of contact residence times: data are presented as time-weighted average values, with $95 \%$ confidence intervals estimated by a $10 \mathrm{k}$ resample bootstrapping of the time-weighted averaging, and the significance level for the difference between binding durations was obtained by a continuitycorrected one-tailed Mann-Whitney-Wilcoxon $U$ test. The Visual Molecular Dynamics (VMD) software ${ }^{67}$ was used to create images and movies.

Reporting summary. Further information on experimental design is available in the Nature Research Reporting Summary linked to this article.

\section{Data availability}

Data supporting the findings of this manuscript are available from the corresponding authors upon reasonable request. A Reporting Summary for this Article is available as a Supplementary Information file. Full scans of gels and blots are provided in Supplementary Information. The source data underlying Figs. 3d, 4b, 5b, 6c, 7b, 7c and Supplementary Table 1 are provided as a Source Data file.

\section{Code availability}

Trajectory analysis programs, as well as protein structures and models, are available from the download section of the Melo lab's website, at http://www.itqb.unl.pt/labs/multiscalemodeling/.

Received: 26 July 2018 Accepted: 22 March 2019

Published online: 23 April 2019

\section{References}

1. Hannun, Y. A. \& Obeid, L. Sphingolipids and their metabolism in physiology and disease. Nat. Rev. Mol. Cell Biol. 19, 175-191 (2018).

2. Holthuis, J. C., Pomorski, T., Raggers, R. J., Sprong, H. \& Van Meer, G. The organizing potential of sphingolipids in intracellular membrane transport. Physiol. Rev. 81, 1689-1723 (2001).
3. Carreira, A. C., Ventura, A. E., Valera, A. R. \& Silva, L. C. Tackling the biophysical properties of sphingolipids to decipher their biological roles. Biol. Chem. 396, 597-609 (2015).

4. Morad, S. A. F. \& Cabot, M. C. Ceramide-orchestrated signalling in cancer cells. Nat. Rev. Cancer 13, 51-65 (2013).

5. Ogretman, B. Sphingolipid metabolism in cancer signaling and therapy. Nat. Rev. Cancer 18, 33-50 (2018).

6. García-Ruiz, C. et al. Defective TNF-alpha-mediated hepatocellular apoptosis and liver damage in acidic sphingomyelinase knockout mice. J. Clin. Invest. 111, 197-208 (2003).

7. Luberto, C. et al. Inhibition of tumor necrosis factor-induced cell death in MCF7 by a novel inhibitor of neutral sphingomyelinase. J. Biol. Chem. 277, 41128-41139 (2002).

8. Deng, X. et al. Ceramide biogenesis is required for radiation-induced apoptosis in the germ line of C. elegans. Science 322, 110-115 (2008).

9. Mesicek, J. et al. Ceramide synthases 2, 5, and 6 confer distinct roles in radiation-induced apoptosis in HeLa cells. Cell Signal. 22, 1300-1307 (2010).

10. Bose, R. et al. Ceramide synthase mediates daunorubicin-induced apoptosis: an alternative mechanism for generating death signals. Cell 82, 405-414 (1995).

11. Alphonse, G. et al. Overcoming resistance to gamma-rays in squamous carcinoma cells by poly-drug elevation of ceramide levels. Oncogene $\mathbf{2 3}$, 2703-2715 (2004)

12. Canals, D. \& Hannun, Y. A. Novel chemotherapeutic drugs in sphingolipid cancer research. Handb. Exp. Pharmacol. 215, 211-239 (2013).

13. Cho, S. M. \& Kwon, H. J. Acid ceramidase, an emerging target for anti-cancer and anti-angiogenesis. Arch. Pharm. Res. https://doi.org/10.1007/s12272-01901114-3 (2019).

14. Tait, S. W. G. \& Green, D. R. Mitochondrial regulation of cell death. Cold Spring Harb. Perspect. Biol. 5, a008706 (2013).

15. Czabotar, P. E., Lessene, G., Strasser, A. \& Adams, J. M. Control of apoptosis by the BCL-2 protein family: implications for physiology and therapy. Nat. Rev. Mol. Cell Biol. 15, 49-63 (2014).

16. Luna-Vargas, M. P. A. \& Chipuk, J. E. The deadly landscape of proapoptotic BCL-2 proteins in the outer mitochondrial membrane. FEBS. J. 283, 2676-2689 (2016).

17. Kuwana, T. et al. Bid, Bax, and lipids cooperate to form supramolecular openings in the outer mitochondrial membrane. Cell 111, 331-342 (2002).

18. Salvador-Gallego, R. et al. Bax assembly into rings and arcs in apoptotic mitochondria is linked to membrane pores. EMBO J. 35, 389-401 (2016).

19. Bourbon, N. A., Sandirasegarane, L. \& Kester, M. Ceramide-induced inhibition of Akt is mediated through protein kinase Czeta: implications for growth arrest. J. Biol. Chem. 277, 3286-3292 (2002).

20. Zhu, Q.-Y. et al. C6-ceramide synergistically potentiates the anti-tumor effects of histone deacetylase inhibitors via AKT dephosphorylation and $\alpha$-tubulin hyperacetylation both in vitro and in vivo. Cell Death Dis. 2, el17 (2011).

21. Dobrowsky, R. T., Kamibayashi, C., Mumby, M. C. \& Hannun, Y. A. Ceramide activates heterotrimeric protein phosphatase 2A. J. Biol. Chem. 268, 15523-15530 (1993).

22. Mukhopadhyay, A. et al. Direct interaction between the inhibitor 2 and ceramide via sphingolipidprotein binding is involved in the regulation of protein phosphatase 2A activity and signaling. FASEB J. 23, 751-763 (2009).

23. Patwardhan, G. A., Beverly, L. J. \& Siskind, L. J. Sphingolipids and mitochondrial apoptosis. J. Bioenerg. Biomembr. 48, 153-168 (2016).

24. Birbes, H. et al. A mitochondrial pool of sphingomyelin is involved in TNFalpha-induced Bax translocation to mitochondria. Biochem. J. 386, 445-451 (2005).

25. Jain, A., Beutel, O., Ebell, K., Korneev, S. \& Holthuis, J. C. M. Diverting CERTmediated ceramide transport to mitochondria triggers Bax-dependent apoptosis. J. Cell Sci. 130, 360-371 (2017).

26. Stiban, J., Caputo, L. \& Colombini, M. Ceramide synthesis in the endoplasmic reticulum can permeabilize mitochondria to proapoptotic proteins. J. Lipid Res. 49, 625-634 (2008).

27. Siskind, L. J., Kolesnick, R. N. \& Colombini, M. Ceramide forms channels in mitochondrial outer membranes at physiologically relevant concentrations. Mitochondrion 6, 118-125 (2006).

28. Colombini, M. Ceramide channels and mitochondrial outer membrane permeability. J. Bioenerg. Biomembr. 49, 57-64 (2016).

29. Siskind, L. J. et al. Anti-apoptotic $\mathrm{Bcl}-2$ family proteins disassemble ceramide channels. J. Biol. Chem. 283, 6622-6630 (2008).

30. Lee, $\mathrm{H}$. et al. Mitochondrial ceramide-rich macrodomains functionalize Bax upon irradiation. PLoS ONE 6, e19783 (2011).

31. Ganesan, V. et al. Ceramide and activated Bax act synergistically to permeabilize the mitochondrial outer membrane. Apoptosis 15, 553-562 (2010).

32. Chipuk, J. E. et al. Sphingolipid metabolism cooperates with BAK and BAX to promote the mitochondrial pathway of apoptosis. Cell 148, 988-1000 (2012). 
33. Naghdi, S., Várnai, P. \& Hajnóczky, G. Motifs of VDAC2 required for mitochondrial Bak import and tBid-induced apoptosis. Proc. Natl. Acad. Sci. USA 112, E5590-E5599 (2015).

34. Lauterwasser, J. et al. The porin VDAC2 is the mitochondrial platform for Bax retrotranslocation. Sci. Rep. 6, 32994 (2016).

35. Chin, H. S. et al. VDAC2 enables BAX to mediate apoptosis and limit tumor development. Nat. Commun. 9, 4976 (2018).

36. Bockelmann, S. et al. A search for ceramide binding proteins using bifunctional lipid analogs yields CERT-related protein StarD7. J. Lipid Res. 59, 515-530 (2018).

37. Nagaraj, N. et al. Deep proteome and transcriptome mapping of a human cancer cell line. Mol. Syst. Biol. 7, 548-548 (2011).

38. Ingólfsson, H. I. et al. Lipid organization of the plasma membrane. J. Am. Chem. Soc. 136, 14554-14559 (2014).

39. Melo, M. N. et al. High-throughput simulations reveal membrane-mediated effects of alcohols on MscL gating. J. Am. Chem. Soc. 139, 2664-2671 (2017).

40. van Eerden, F. J., Melo, M. N., Frederix, P. W. J. M., Periole, X. \& Marrink, S. J. Exchange pathways of plastoquinone and plastoquinol in the photosystem II complex. Nat. Commun. 8, 15214 (2017).

41. Choudhary, O. P. et al. Structure-guided simulations illuminate the mechanism of ATP transport through VDAC1. Nat. Struct. Mol. Biol. 21, 626-632 (2014).

42. Schredelseker, J. et al. High resolution structure and double electron-electron resonance of the zebrafish voltage-dependent anion channel 2 reveal an oligomeric population. J. Biol. Chem. 289, 12566-12577 (2014).

43. Horvath, S. E. \& Daum, G. Lipids of mitochondria. Prog. Lipid. Res. 52, 590-614 (2013).

44. Weiser, B. P., Salari, R., Eckenhoff, R. G. \& Brannigan, G. Computational investigation of cholesterol binding sites on mitochondrial VDAC. J. Phys. Chem. B 118, 9852-9860 (2014).

45. Villinger, S. et al. Functional dynamics in the voltage-dependent anion channel. Proc. Natl. Acad. Sci. USA 107, 22546-22551 (2010).

46. Budelier, M. M. et al. Photoaffinity labeling with cholesterol analogues precisely maps a cholesterol-binding site in voltage-dependent anion channel1. J. Biol. Chem. 292, 9294-9304 (2017).

47. Das, J. Aliphatic diazirines as photoaffinity probes for proteins: recent developments. Chem. Rev. 111, 4405-4417 (2011).

48. De Pinto, V., Al Jamal, J. A. \& Palmieri, F. Location of the dicyclohexylcarbodiimide-reactive glutamate residue in the bovine heart mitochondrial porin. J. Biol. Chem. 268, 12977-12982 (1993).

49. Keinan, N., Tyomkin, D. \& Shoshan-Barmatz, V. Oligomerization of the mitochondrial protein voltage-dependent anion channel is coupled to the induction of apoptosis. Mol. Cell. Biol. 30, 5698-5709 (2010).

50. Huang, L. et al. A new fungal diterpene induces VDAC1-dependent apoptosis in Bax/Bak-deficient cells. J. Biol. Chem. 290, 23563-23578 (2015).

51. Bergdoll, L. A. et al. Protonation state of glutamate 73 regulates the formation of a specific dimeric association of mVDAC1. Proc. Natl. Acad. Sci. USA 115, E172-E179 (2018).

52. Zhou, K. et al. A ceramide-regulated element in the late endosomal protein LAPTM4B controls amino acid transporter interaction. ACS Cent. Sci. 4, 548-558 (2018).

53. Renault, T. T. et al. Mitochondrial shape governs BAX-induced membrane permeabilization and apoptosis. Mol. Cell 57, 69-82 (2015).

54. Zaid, H., Abu-Hamad, S., Israelson, A., Nathan, I. \& Shoshan-Barmatz, V. The voltage-dependent anion channel-1 modulates apoptotic cell death. Cell Death Differ. 12, 751-760 (2005).

55. Abu-Hamad, S., Zaid, H., Israelson, A., Nahon, E. \& Shoshan-Barmatz, V. Hexokinase-I protection against apoptotic cell death is mediated via interaction with the voltage-dependent anion channel-1: mapping the site of binding. J. Biol. Chem. 283, 13482-13490 (2008).

56. Shoshan-Barmatz, V., Ben-Hail, D., Admoni, L., Krelin, Y. \& Tripathi, S. S. The mitochondrial voltage-dependent anion channel 1 in tumor cells. Biochim. Biophys. Acta 1848, 2547-2575 (2015).

57. Mathupala, S. P., Ko, Y. H. \& Pedersen, P. L. Hexokinase II: cancer's doubleedged sword acting as both facilitator and gatekeeper of malignancy when bound to mitochondria. Oncogene 25, 4777-4786 (2006).

58. Wieczorek, H. et al. Isolation of goblet cell apical membrane from tobacco hornworm midgut and purification of its vacuolar-type ATPase. Methods Enzymol. 192, 608-616 (1990).

59. Wassenaar, T. A., Ingólfsson, H. I., Böckmann, R. A., Tieleman, D. P. \& Marrink, S. J. Computational lipidomics with insane: a versatile tool for generating custom membranes for molecular simulations. J. Chem. Theory Comput. 11, 2144-2155 (2015).

60. Marrink, S. J., Risselada, H. J., Yefimov, S., Tieleman, D. P. \& de Vries, A. H. The MARTINI force field: coarse grained model for biomolecular simulations. J. Phys. Chem. B 111, 7812-7824 (2007).
61. Periole, X., Cavalli, M., Marrink, S.-J. \& Ceruso, M. A. Combining an elastic network with a coarse-grained molecular force field: structure, dynamics, and intermolecular recognition. J. Chem. Theory Comput. 5, 2531-2543 (2009).

62. Hess, B., Kutzner, C., van der Spoel, D. \& Lindahl, E. GROMACS 4: algorithms for highly efficient, load-balanced, and scalable molecular simulation. J. Chem. Theory Comput. 4, 435-447 (2008).

63. de Jong, D. H. et al. Improved parameters for the Martini coarse-grained protein force field. J. Chem. Theory Comput. 9, 687-697 (2013).

64. Michaud-Agrawal, N., Denning, E. J., Woolf, T. B. \& Beckstein, O. MDAnalysis: a toolkit for the analysis of molecular dynamics simulations. $J$. Comput. Chem. 32, 2319-2327 (2011).

65. Gowers, R. J., et al. MDAnalysis: a Python package for the rapid analysis of molecular dynamics simulations. In Proc. of the 15th Python in Science Conference (eds. Benthall, S. \& Rostrup, S.) 98-105, Austin, TX (SciPy, 2016).

66. van der Walt, S., Colbert, S. C. \& Varoquaux, G. The NumPy array: a structure for efficient numerical computation. Comput. Sci. Eng. 13, 22-30 (2011).

67. Humphrey, W., Dalke, A. \& Schulten, K. VMD: visual molecular dynamics. $J$. Mol. Graph. 14, 33-8-27-8 (1996).

\section{Acknowledgements}

This work was supported by the Deutsche Forschungsgemeinschaft (Projects SFB944P14 and HO3539/1-1) to J.C.M.H., a Marie Curie Intra-European Fellowship to J.G.M.M (Project 289278), and a German Egyptian Research Long-term Scholarship to D.G.H. (Project 57222240). M.N.M was supported by Project LISBOA-01-0145-FEDER-007660 (Microbiologia Molecular, Estrutural e Celular) funded by FEDER funds through COMPETE2020-Programa Operacional Competitividade e Internacionalização (POCI) and by national funds through FCT-Fundação para a Ciência e a Tecnologia. M.N.M. and G.R. acknowledge the National Laboratory for Scientific Computing (LNCC/MCTI, Brazil) for providing HPC resources of the SDumont supercomputer (http://sdumont. lncc.br). Additional support was provided by the NIH (Project 1R21AI124225-01A1) to F.G.T.

\section{Author contributions}

J.C.M.H. conceptualized the study and wrote the manuscript, with critical input from S.D., S.B., J.G.M.M., and M.N.M. J.G.M.M. designed and performed the photolabeling, fractionation, purification, and identification of ceramide-binding proteins. M.N.M. designed, performed, and analyzed the computer simulation studies, with critical input from G.R. and S.J.M. S.K. synthesized photoactive and clickable lipid analogs. S.B., D.G.H., H.J., D.M., and M.S. designed and performed the reconstitution and photolabeling of recombinant protein channels. S.D. and P.N. created and characterized mutant cell lines, with critical input from F.G.T. S.D. designed and performed transfection studies and photolabeling of purified mitochondria, with critical input from D.G.H.

\section{Additional information}

Supplementary Information accompanies this paper at https://doi.org/10.1038/s41467 019-09654-4.

Competing interests: The authors declare no competing interests.

Reprints and permission information is available online at http://npg.nature.com/ reprintsandpermissions/

Journal peer review information: Nature Communications thanks Alex Evers, Oliver Beckstein, and the other anonymous reviewer(s) for their contribution to the peer review of this work. Peer reviewer reports are available.

Publisher's note: Springer Nature remains neutral with regard to jurisdictional claims in published maps and institutional affiliations.

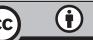

Open Access This article is licensed under a Creative Commons Attribution 4.0 International License, which permits use, sharing, adaptation, distribution and reproduction in any medium or format, as long as you give appropriate credit to the original author(s) and the source, provide a link to the Creative Commons license, and indicate if changes were made. The images or other third party material in this article are included in the article's Creative Commons license, unless indicated otherwise in a credit line to the material. If material is not included in the article's Creative Commons license and your intended use is not permitted by statutory regulation or exceeds the permitted use, you will need to obtain permission directly from the copyright holder. To view a copy of this license, visit http://creativecommons.org/ licenses/by/4.0/.

(C) The Author(s) 2019 